\title{
Constructing all-order corrections to multi-jet rates
}

\section{Jeppe R. Andersen ${ }^{a}$ and Jennifer M. Smillie ${ }^{b}$}

${ }^{a}$ Theory Division, Physics Department, CERN, CH-1211 Geneva 23, Switzerland

${ }^{b}$ IPPP, University of Durham, Durham, DH1 3LE, U.K.

E-mail: jeppe.andersen@cern.ch, smillie@hep.ucl.ac.uk

ABSTRACT: We discuss the universal behaviour of scattering cross sections in the limit of infinite rapidity separation between all produced particles, and illustrate the behaviour explicitly for the production of $n$ jets, $W+n$ jets, $Z+n$ jets for $n=2,3,4$, and for $H+2,3$ jets. We give a set of rules for constructing scattering cross sections, which are exact in the given limit, and order-by-order reproduce well the full fixed order results when applied to LHC phenomenology. The approximation includes both real and virtual corrections, and is sufficiently simple to allow for the regulated all-order perturbative sum to be explicitly constructed. We test the expected accuracy by comparing results orderby-order with full fixed-order perturbation theory for the processes mentioned above.

KEYwords: Jets, Hadronic Colliders, QCD

ARXIV EPRINT: 0908.2786 


\section{Contents}

1 Introduction 1

2 The high energy limit of scattering amplitudes 3

3 The method of currents $\quad 7$

3.1 Current-current scattering 8

$\begin{array}{lll}3.2 & \text { Multi-parton production } & 10\end{array}$

$\begin{array}{lll}3.3 & \mathrm{~W} \text { and } \mathrm{Z} \text { boson production in association with jets } & 13\end{array}$

$\begin{array}{ll}3.4 \text { Higgs boson production in association with jets } & 16\end{array}$

$\begin{array}{ll}3.5 & \text { Virtual corrections and regularisation } \\ \end{array}$

4 Applications $\quad 20$

$\begin{array}{lll}4.1 & \text { Pure jets } & 21\end{array}$

$4.2 \mathrm{~W}+$ jets 22

$\begin{array}{lll}4.3 \mathrm{Z}+\text { jets } & 26\end{array}$

$\begin{array}{lll}4.4 & \text { Higgs boson }+ \text { jets } & 27\end{array}$

$\begin{array}{lll}5 & \text { Conclusions } & 30\end{array}$

$\begin{array}{ll}\text { A Spinor representation } & 31\end{array}$

B Momentum configurations $\quad 32$

\section{Introduction}

Achieving the full potential of the LHC in furthering our understanding of particle physics will challenge our understanding and description of events with multiple jets. Such events arise both within the Standard Model (SM), and in many models of physics beyond the SM. Apart from furthering our understanding of the solutions to the SM equations of motion, a better understanding and description of the multi-jet predictions arising from the SM is necessary in order to fully disentangle this contribution from that which might arise from outside the SM.

The true complication of an observed jet in terms of its constituent hadrons can currently only be described within the context of a "General Purpose Monte Carlo", implementing a parton shower and hadronisation model as described in e.g. [1-3]. The parton shower is based on a resummation of soft and collinear radiation. However, while obtaining a good description of the structure of each jet, this description severely underestimates the rate and hardness ( $p_{\perp}$-spectrum) of multi-jet samples. Better predictions of such quantities in exclusive (meaning fixed number) jet samples have so far been obtained in fixed order 
calculations; for processes allowing more than two light partons in the final state, such predictions are currently limited to NLO accuracy. However, putting aside issues of hadronisation, many other questions which are important for the LHC programme still cannot be answered satisfactorily in the relatively simple description of the final states obtained at such low orders in the perturbative expansion. Such questions range from the effects of central jet vetos in samples of Higgs boson plus jets, to the simple question of the transverse momentum spectrum of weak gauge bosons (both at large and small transverse momenta) or the relative weight of various jet multiplicities in inclusive samples ("inclusive" here meaning sum over any number of jets).

A combination of fixed order calculations and further parton shower resummation has been achieved, which so far corrects the approximation obtained in the parton shower to full tree-level accuracy (for a fixed jet multiplicity $[4,5]$ ) or NLO accuracy $[6,7]$ (so far in processes which at LO have just zero or one light parton in the final state). Such matching schemes ensure that a given process is described correctly at least at (N)LO, while all higher order corrections are estimated from the parton shower.

All resummation schemes are built upon a particular kinematic limit in which the perturbative corrections simplify, allowing for all-order approximations to the full perturbative series. For the parton shower, this is the soft and the collinear limit, i.e. emissions under small invariant mass, and the building blocks are e.g. parton splitting functions. In the current study, we will be guided by the simplifications of the perturbative corrections in the case of large invariant mass between all emissions. We will seek to catch the part of the perturbative corrections which controls additional jet production, but ignore the collinear behaviour which gives rise to the jet-sub-structure. We will obtain an approximation to both virtual and real-emission corrections, which allows us to build an approximation to the regularised, all-orders matrix element for each exclusive (resolved) parton multiplicity. The focus of this paper is to obtain the relevant universal building blocks for multi-jet predictions. Furthermore, we will investigate the accuracy of this approach by comparing the lowest order predictions obtained in our simplified approach to the full tree-level QCD perturbative series for $n$-jet-production, $(n=2,3,4)$, both "pure" and in association with a $W, Z$ or Higgs-boson.

The approximate or " $t$-channel factorised" matrix element will be sufficiently fast to evaluate numerically that the resummation can be constructed by explicit summation over the exclusive final states, and thus any event analysis can be performed by simply imposing jet algorithms etc. on the exclusive final states ("exclusive" meaning fully differential in the momenta of all produced particles). We will leave the phenomenological implications of the resummation of each of the four processes discussed here to future studies.

In section 2 we will introduce the Multi-Regge-Kinematic-limit, which lies at the heart of our approach, and discuss and illustrate the universal behaviour of scattering amplitudes in this limit. In section 3 we will construct all the building blocks for the relevant amplitudes. In section 4 we will check process-by-process, multiplicity-by-multiplicity the level of accuracy at which the approach reproduces the fixed order perturbative expansion in terms of not just cross sections but also differential distributions, where these can be obtained with standard tools implementing the full tree-level processes. By doing so, we 


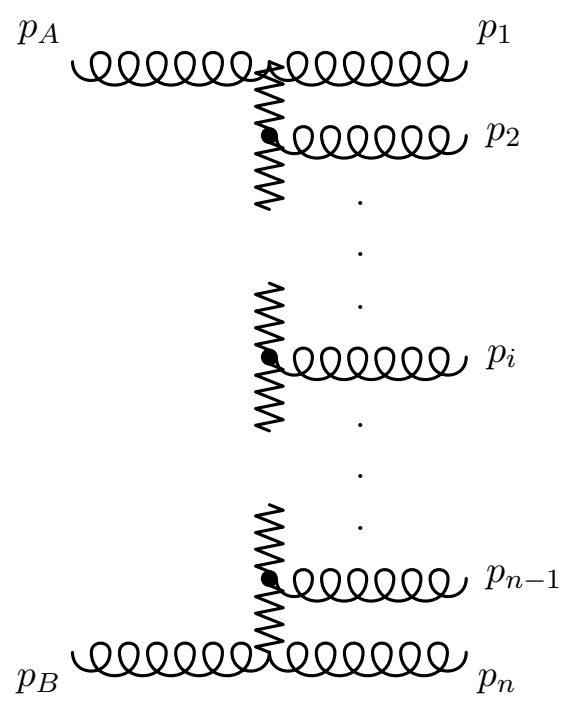

Figure 1. Picture of effective vertices connected by $t$-channel exchanges.

hope to instill trust in the all-order approximation which can be built using the elements described in this paper.

\section{The high energy limit of scattering amplitudes}

We will start by studying the scattering matrix elements of a gluonic $2 \rightarrow n, n \geq 2$, process in the so-called Multi-Regge-Kinematic (MRK) limit, where the scattering momenta $p_{A}, p_{B} \rightarrow p_{1}, \ldots, p_{n}$ in terms of transverse momenta and rapidity $y=\ln \left(\frac{E+p_{z}}{E-p_{z}}\right)$ fulfil the following conditions

$$
\begin{array}{r}
\forall i \in\{2, \ldots, n-1\}: y_{i-1} \gg y_{i} \gg y_{i+1} \\
\forall i, j:\left|p_{i \perp}\right| \approx\left|p_{j \perp}\right|,
\end{array}
$$

or alternatively

$$
\forall i, j:\left|p_{i \perp}\right| \approx\left|p_{j \perp}\right|, s_{i j} \rightarrow \infty
$$

where $s_{i j}=2 p_{i} \cdot p_{j}$ and $s=2 p_{A} \cdot p_{B}$. The notation $y_{i} \gg y_{j}$ really means $y_{i}-y_{j} \rightarrow \infty$. The transverse directions are with respect to the incoming partons $p_{A}, p_{B}$ (i.e. transverse to the beam line), and the limit requires the transverse components to be kept fixed (i.e. not growing with $s$ ) as $s_{i j},\left|y_{i}-y_{j}\right| \rightarrow \infty$. We can take $p_{A}$ to be the direction of positive light-cone momentum.

Explicit calculations of up to $2 \rightarrow 4$ gluon scattering [8], and a dispersive analysis of the $2 \rightarrow n$ process $[9,10]$ showed that in the MRK limit, the $2 \rightarrow n$ scattering amplitude is dominated by the behaviour dictated by the poles from $t$-channel gluon exchanges. $t$ channel here refers to the picture which arises of a string of effective vertices for on-shell gluon production, which are connected by off-shell ( $t$-channel) gluon currents, see figure 1 . The $t$-channel momenta are here defined as

$$
t_{i}=p_{A}-\sum_{j=1}^{i} p_{j} .
$$


In section 3 we will illustrate how the following result arises, and re-derive the form of the building blocks of the approximation. Here we will just quote the result for the LO colour and spin summed and averaged scattering matrix element for $2 \rightarrow n$ on-shell gluons in the MRK limit:

$$
\left|\overline{\mathcal{M}}_{g g \rightarrow g \cdots g}^{M R K}\right|^{2}=\frac{4 s^{2}}{N_{C}^{2}-1} \frac{g^{2} C_{A}}{\left|p_{1 \perp}\right|^{2}}\left(\prod_{i=2}^{n-1} \frac{4 g^{2} C_{A}}{\left|p_{i \perp}\right|^{2}}\right) \frac{g^{2} C_{A}}{\left|p_{n \perp}\right|^{2}}
$$

One notices that obviously, after the MRK limit is taken, there is no dependence left on the rapidities of the gluons (or rather, the dependence on the rapidities is lost). The dependence on the centre-of-mass energy $s$ in eq. (2.4) is left in, although of course in the MRK limit, $s \rightarrow \infty$. The partonic cross section is found by dividing the square of the scattering matrix element by the flux factor, which is proportional to $s^{2}$, so the MRK limit of the partonic cross section is a very simple function of only the transverse momenta of the emitted gluons.

The results of ref. [9-11] extend beyond just pure gluon scattering; the picture which arises is one in which the description of a given $2 \rightarrow n$ scattering process in the MRK limit factorises into a product of effective vertices for particle emission, with the vertices connected by propagators according to the ordering in rapidity of the emitted particles. One immediate result is that in the MRK limit, the $2 \rightarrow n$ scattering process is dominated by rapidity orderings which allow pure gluon exchanges (or more precisely: exchanges of the particle of highest spin) between the scattering vertices.

Another immediate result of the factorisation (arising from the assumption of a hierarchy in the components of plus- and minus-momenta) is that in the MRK limit, the scattering matrix element for e.g. $g g \rightarrow g g g, q g \rightarrow q g g$ and $q Q \rightarrow q g Q$ differ only by colour factors - this is a generalisation of the results leading to the "effective PDF"-approach to $2 \rightarrow 2$ scattering [12]. The results equivalent to eq. (2.4) for the $q Q$ and $q g$-initiated scattering processes in the MRK limit are then

$$
\begin{gathered}
\left|\overline{\mathcal{M}}_{q g \rightarrow q g \cdots g}^{M R K}\right|^{2}=\frac{4 s^{2}}{N_{C}^{2}-1} \frac{g^{2} C_{F}}{\left|p_{1 \perp}\right|^{2}}\left(\prod_{i=2}^{n-1} \frac{4 g^{2} C_{A}}{\left|p_{i \perp}\right|^{2}}\right) \frac{g^{2} C_{A}}{\left|p_{n \perp}\right|^{2}}, \\
\left|\overline{\mathcal{M}}_{q Q \rightarrow q g \cdots Q}^{M R K}\right|^{2}=\frac{4 s^{2}}{N_{C}^{2}-1} \frac{g^{2} C_{F}}{\left|p_{1 \perp}\right|^{2}}\left(\prod_{i=2}^{n-1} \frac{4 g^{2} C_{A}}{\left|p_{i \perp}\right|^{2}}\right) \frac{g^{2} C_{F}}{\left|p_{n \perp}\right|^{2}},
\end{gathered}
$$

with the additional information that all final states not ordered in rapidity according to the indication in the subscripts are suppressed (by powers of $s_{i j}$ ). The difference in colour factor when gluon 1 or $n$ is replaced by a quark is $C_{F} / C_{A}$, since the difference in the summed and averaged colour factor is the replacement of $f^{a b c} f^{a b d} /\left(N_{C}^{2}-1\right)=C_{A} \delta^{c d} /\left(N_{C}^{2}-1\right)$ with $t_{i j}^{c} t_{j i}^{d} / N_{C}=\delta^{c d} /\left(2 N_{C}\right)$. Eqs. (2.4)-(2.6) arise also as the expansions to fixed order of the solution to the BFKL equation to leading logarithmic accuracy.

The results of eqs. (2.4)-(2.6) are summarised in figure 2 , which illustrates the be- 


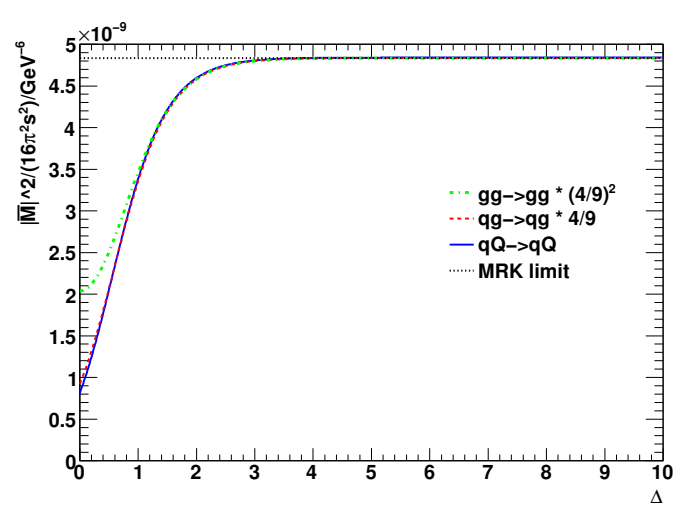

(a)

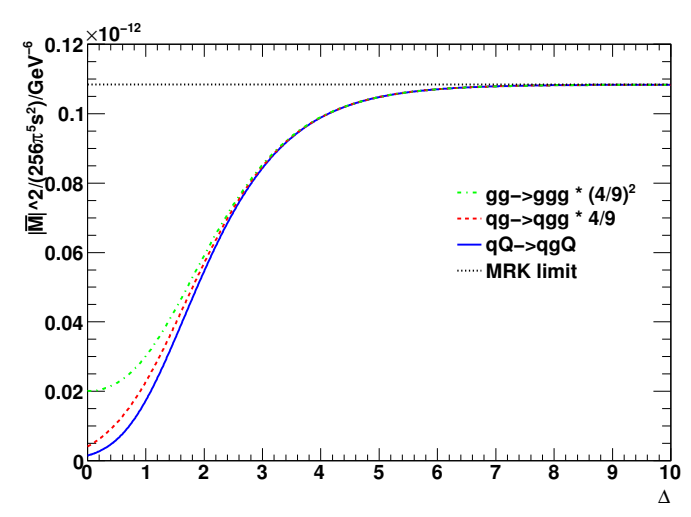

(b)

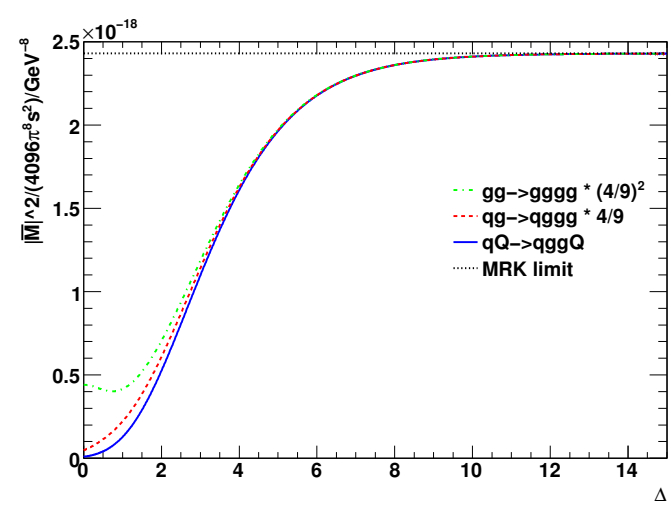

(c)

Figure 2. The scattering matrix elements for (a) $q Q \rightarrow q Q, q g \rightarrow q g$ and $g g \rightarrow g g$, (b) $q Q \rightarrow q g Q$, $q g \rightarrow q g g$ and $g g \rightarrow g g g$ and (c) $q Q \rightarrow q g g Q, q Q \rightarrow q g g Q$ and $g g \rightarrow g g g g$, as a function of the rapidity difference $\Delta$ between the partons, defined in eq. (2.8). The MRK limit is the value obtained from eq. (2.6).

haviour of the quantities
(a) $\frac{|\mathcal{M}|^{2}}{16 \pi^{2} s^{2}} \quad$ for $\quad|\mathcal{M}|^{2} \in\left\{\left(\frac{C_{F}}{C_{A}}\right)^{2}\left|\overline{\mathcal{M}}_{g g \rightarrow g g}\right|^{2},\left(\frac{C_{F}}{C_{A}}\right)\left|\overline{\mathcal{M}}_{q g \rightarrow q g}\right|^{2},\left|\overline{\mathcal{M}}_{q Q \rightarrow q Q}\right|^{2}\right\}$
(b) $\frac{|\mathcal{M}|^{2}}{256 \pi^{5} s^{2}} \quad$ for $\quad|\mathcal{M}|^{2} \in\left\{\left(\frac{C_{F}}{C_{A}}\right)^{2}\left|\overline{\mathcal{M}}_{g g \rightarrow g g g}\right|^{2},\left(\frac{C_{F}}{C_{A}}\right)\left|\overline{\mathcal{M}}_{q g \rightarrow q g g}\right|^{2},\left|\overline{\mathcal{M}}_{q Q \rightarrow q g Q}\right|^{2}\right\}$,

and

(c) $\frac{|\mathcal{M}|^{2}}{4096 \pi^{8} s^{2}} \quad$ for $\quad|\mathcal{M}|^{2} \in\left\{\left(\frac{C_{F}}{C_{A}}\right)^{2}\left|\overline{\mathcal{M}}_{g g \rightarrow g g g g}\right|^{2},\left(\frac{C_{F}}{C_{A}}\right)\left|\overline{\mathcal{M}}_{q g \rightarrow q g g g}\right|^{2},\left|\overline{\mathcal{M}}_{q Q \rightarrow q g g Q}\right|^{2}\right\}$.

The full tree-level matrix elements are extracted from MadGraph [13], and the momenta 


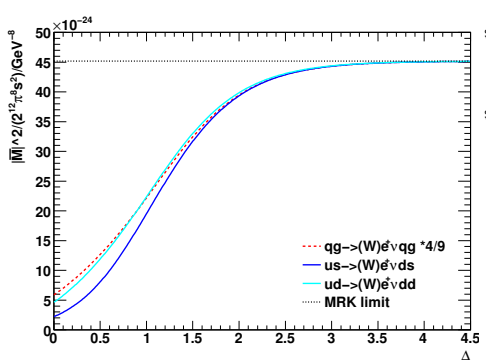

(a)

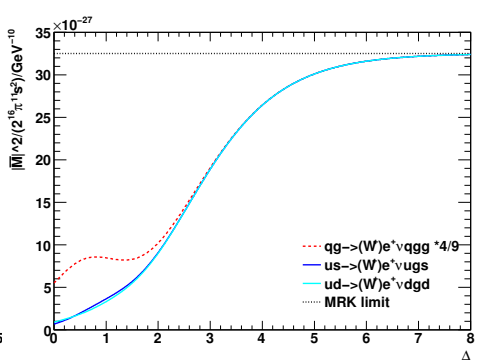

(b)

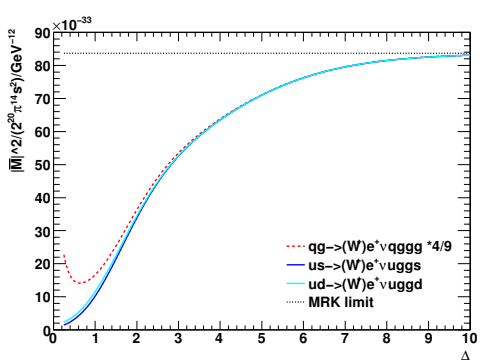

(c)

Figure 3. The scattering matrix elements for jet production in association with a $W$ boson, for (a) 2, (b) 3 and (c) 4 jets.
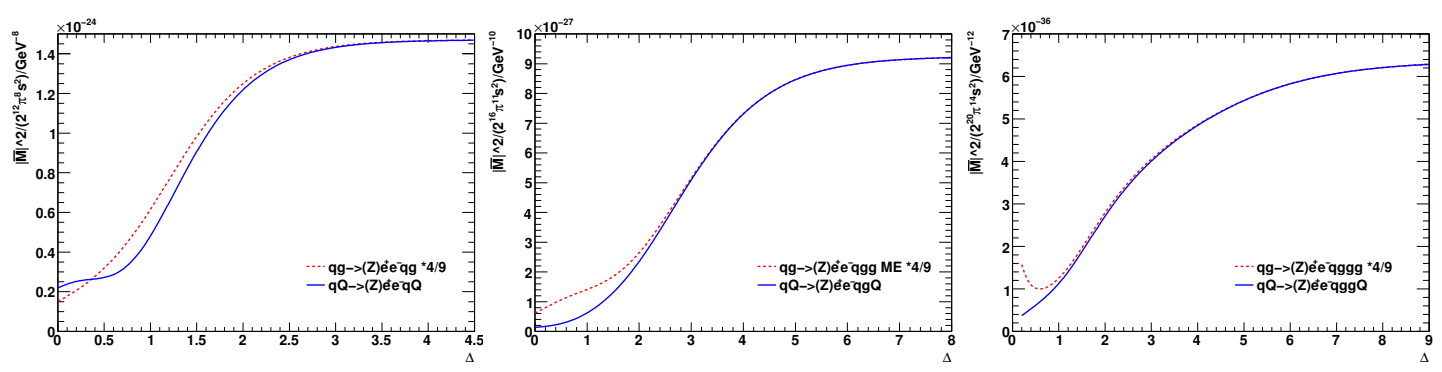

Figure 4. The scattering matrix elements for jet production in association with a $Z$ boson, for (a) 2 , (b) 3 and (c) 4 jets.

of the final state particles are chosen as

$$
p_{i}=\left(k_{\perp} \cosh \left(y_{i}\right), k_{\perp} \cos \left(\phi_{i}\right), k_{\perp} \sin \left(\phi_{i}\right), k_{\perp} \sinh \left(y_{i}\right)\right), k_{\perp}=40 \mathrm{GeV}
$$

(a) : $y_{1}=\Delta, y_{2}=-\Delta, \quad \phi_{1}=0, \phi_{2}=\pi$,

(b) : $y_{1}=\Delta, y_{2}=0, y_{3}=-\Delta, \quad \phi_{1}=0, \phi_{2}=\frac{2 \pi}{3}, \phi_{3}=-\frac{2 \pi}{3}$,

(c) : $y_{1}=\Delta, y_{2}=\frac{\Delta}{3}, y_{3}=-\frac{\Delta}{3}, y_{4}=-\Delta, \quad \phi_{1}=0, \phi_{2}=\frac{\pi}{2}, \phi_{3}=-\frac{\pi}{2}, \phi_{4}=\pi$.

The same universal behaviour is seen in processes where a $W, Z$ or $H$ boson is produced in association with jets, as shown in figures $3-5$. The momentum configurations for these plots are given in appendix B. The rapidities of the jets in each case are as in eq. (2.8), except for the $H+3 j$ process, where the jet rapidities are $\Delta,-\Delta / 3$ and $-\Delta$ respectively. The MRK limit for the $W$ processes is taken from ref. [14] while that for the Higgs processes is taken from ref. [15].

As can be seen on the plots, the correct MRK limit is obtained for all the channels. However, while the simple formulae of eqs. (2.4)-(2.6) do indeed describe the MRK limit of the full amplitudes, this limit is approached only outside the region of relevance for the LHC. The power vested in this implementation of the factorised picture in terms of ability to calculate a specific limit of the $n$-gluon amplitude is turning out not to be relevant for LHC phenomenology, if implemented according to eq. (2.4) or the BFKL equation. 


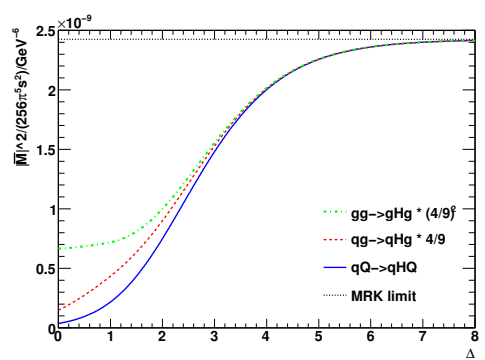

(a)

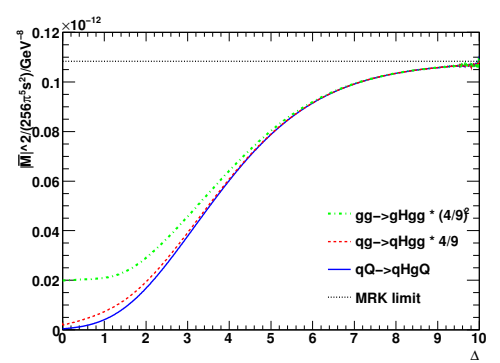

(b)

Figure 5. The scattering matrix elements for jet production in association with a $H$ boson, for (a) 2 and (b) 3 jets.

In contrast, the universality of the $q Q, q g$ and $g g$-channels, which arises naturally within and to some extent even implies the picture indicated in figure 1, is reached in the region relevant for the LHC phenomenology. In fact, the $q Q$ and $q g$ channels behave very similarly over a very wide range of rapidities. This poses the question whether one can construct better building blocks still within a factorised picture, which would allow one to get estimates for the all-order cross sections, but crucially in a wider kinematic region which would be relevant for the LHC. This is the focus of the next section.

\section{The method of currents}

The aim of this section is to construct a method for approximating the hard scattering matrix element for jet production (pure or in association with a $W / Z / H$-boson) to any order in $\alpha_{s}$. The method should have the following characteristics:

1. Inclusivenes: The approach should capture both real and virtual corrections to all orders for a given process.

2. Simplicity:

(a) All orders exclusively: In order to allow for arbitrary analyses of the process, the all-order result must be constructed as an explicit sum over $n$-particle final states, with access to the momenta of all emitted particles. This obviously requires that the evaluation of the scattering amplitude for any number (necessary) of particles is sufficiently fast to allow for the $n$-body phase space integration to be performed explicitly.

(b) Cancellation of IR poles: The formalism has to be sufficiently simple that the cancellation of IR poles between real and virtual corrections can be organised while keeping the all-order summation simple.

3. Accuracy:

(a) The obtained results must reproduce the full perturbative result order by order in $\alpha_{s}$ in the limit of infinite invariant mass between all partons. 

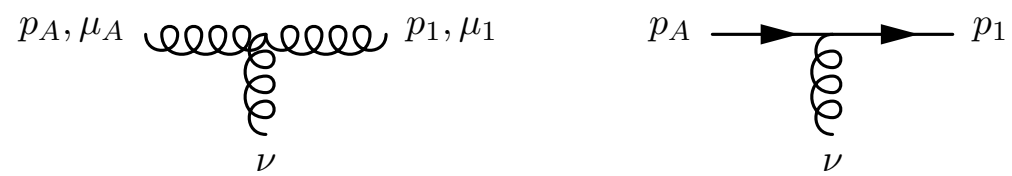

Figure 6. The three-gluon vertex and the quark current shown are identical in the limit $p_{A} \sim p_{1}$, eq. (3.1).

(b) At the same time, the result must maintain relevance in the kinematic regime of $\mathrm{TeV}$-scale colliders; we will calculate the cross section and kinematic distributions for 2,3,4-jet production alone and in association with a $W, Z$ or $H$ boson within the simple procedure allowing an all-order construction, and compare the results to those obtained with full, fixed-order calculations.

Our approach will be based on capturing the behaviour dictated by poles in the $t$-channel momenta of the scattering amplitude (as defined in eq. (2.3), where the momenta $p_{i}$ are rapidity ordered). Furthermore, in this paper we will focus on the rapidity ordering of particles which allows colour octet exchanges between all neighbouring (in rapidity) particles. It is well-known $[10,16,17]$ that for a given $2 \rightarrow n$ process, these rapidity-orderings will form the leading contribution to $n$-jet production, and all other orderings will be suppressed by powers of the invariant mass between jets of flavours which cannot be connected by a colour octet exchange. The relevance of this property of the scattering amplitudes for inclusive cross sections (i.e. not just as an asymptotic argument) was investigated for Higgs boson production in association with at least two jets in ref. [18, 19].

\subsection{Current-current scattering}

Based on the close resemblance between the various partonic channels in $2 \rightarrow 2,2 \rightarrow$ $3,2 \rightarrow 4$ scattering, we choose to model all channels on $q Q \rightarrow q(g \ldots g) Q$-scattering. The similarity in the behaviour of the various partonic channels in the MRK limit, where the $t$-channel dominance has set in, is further supported by the behaviour of the three-gluon vertex and the quark current (figure 6) in the limit $p_{A} \sim p_{1}$ (using the standard spinor formalism):

$$
\begin{aligned}
& \langle 1|\nu| A\rangle \rightarrow 2 p_{A / 1}^{\nu} \\
& \varepsilon_{A \mu_{A}} \varepsilon_{1 \mu_{1}}^{*}\left(\left(p_{A}+p_{1}\right)^{\nu} g^{\mu_{A} \mu_{1}}+\left(q-p_{1}\right)^{\mu_{A}} g^{\mu_{1} \nu}+\left(-q-p_{A}\right)^{\mu_{1}} g^{\mu_{A} \nu}\right) \rightarrow \varepsilon_{A} \cdot \varepsilon_{1}^{*} \times 2 p_{A / 1}^{\nu} .
\end{aligned}
$$

The building blocks of the traditional (B)FKL formalism are termed impact factors (describing the coupling of e.g. two on-shell partons to an off-shell ( $t$-channel) gluon) and the kernel (describing the evolution of the off-shell gluon under the emission of partons). These building blocks can be obtained by taking the MRK limit of specific processes, see e.g. ref. [20] and references therein. However, with building blocks obtained in this way, the resulting approximation for the scattering amplitude will have lost all dependence on the rapidity difference between particles (since this has been taken to infinity), and only the limits in figure 2 are obtained. When the BFKL equation is used, this approximation 
is then applied in all of phase space, resulting in a gross over-estimate order-by-order of the scattering amplitude.

Instead of relying on a kinematic limit to ensure the $t$-channel dominance of the scattering, we will instead obtain the building blocks from the process $q Q \rightarrow q Q$, which consists only of a $t$-channel gluon exchange. This means that the lowest order results for the $q Q \rightarrow q Q$ process will be identical in full QCD and with the effective rules. The benefits are even greater for e.g. W or Z plus jets, where in the standard approach (see e.g. ref. [14]), kinematical assumptions on also the weak boson (and its decay products) as well as the jets had to be applied in order to obtain the building blocks for a resummation. We will see that the results for Higgs boson production in association with jets $[15,18,19]$ can also be improved using this new formalism.

When the effective Feynman rules we derive here are used in constructing all-order results, the differences between the various partonic channels at small rapidities (and the corrections to the approximations for multi-parton production) will be taken into account order-by-order by matching to the full fixed-order perturbative result using the same approach as in ref. [18, 19].

In the spinor notation, the (colour and coupling stripped) matrix element for the process $q Q \rightarrow q Q$ for negative helicity quarks reads

$$
\begin{aligned}
M_{q^{-} Q^{-} \rightarrow q^{-} Q^{-}} & =\langle 1|\mu| a\rangle \frac{g^{\mu \nu}}{t}\langle 2|\nu| b\rangle \\
& =\frac{2[a b]\langle 21\rangle}{t} .
\end{aligned}
$$

We will use the scattering of quark currents as the basis for our framework, since these consist of $t$-channel gluon exchanges only, and explicitly exhibit the factorisation into two components (spinor strings), each depending only on the momenta along each quark line. This is also obviously true for all other helicity configurations.

Let us denote the spinor string (for helicities $h_{a}, h_{1}, h_{b}, h_{2}$ of the quarks) appearing in the amplitude as

$$
S_{q Q \rightarrow q Q}^{h_{a} h_{b} \rightarrow h_{1} h_{2}}=\left\langle 1 h_{1}|\mu| a h_{a}\right\rangle g^{\mu \nu}\left\langle 2 h_{2}|\nu| b h_{b}\right\rangle
$$

This complex number will be calculated using an explicit representation for the spinors (see appendix A), and we will denote the sum over helicities of the absolute square of this number by

$$
\left\|S_{q Q \rightarrow q Q}\right\|^{2}=\sum_{h_{a}, h_{a}, h_{b}, h_{2}}\left|S_{q Q \rightarrow q Q}^{h_{a} h_{b} \rightarrow h_{1} h_{2}}\right|^{2}
$$

Of course in this case non-zero contributions arise only when $h_{a}=h_{1}$ and $h_{b}=h_{2}$.

The colour and helicity summed and averaged matrix element for the scattering process 
$q Q \rightarrow q Q$ is then

$$
\begin{aligned}
\left|\overline{\mathcal{M}}_{q Q \rightarrow q Q}^{t}\right|^{2}= & \frac{1}{4\left(N_{C}^{2}-1\right)}\left\|S_{q Q \rightarrow q Q}\right\|^{2} \\
& \cdot\left(g^{2} C_{F} \frac{1}{t_{1}}\right) \\
& \cdot\left(g^{2} C_{F} \frac{1}{t_{2}}\right) .
\end{aligned}
$$

with $t_{1}=\left(p_{a}-p_{1}\right)^{2}$ and $t_{2}=\left(-p_{b}+p_{2}\right)^{2}\left(t_{1}=t_{2}\right.$ in this case of a $2 \rightarrow 2$-process $)$. The superscript $t$ is meant to indicate that this is the " $t$-channel factorised"-approximation, but this is of course exact in the channel $q Q \rightarrow q Q$. The approximations for the gluon channels are obtained by multiplying $C_{A} / C_{F}$ for each pair of quarks replaced by gluons. Figure 2 shows that this is an extremely good approximation.

The spinor formalism clearly displays the factorisation in the $t$-channel of the scattering, i.e. within the spinor formalism, the "impact factors" are clearly identified as the quark currents; in contrast, the "spinor product" rewriting of eq. (3.3) mixes momenta from the two quark lines. The standard procedure for extracting impact factors using the helicity formalism [20] applies the kinematic approximations valid in the MRK limit. In terms of invariants, the square of the colour and spin averaged and summed scattering matrix element for $q Q \rightarrow q Q$ is

$$
g^{4} \frac{4}{9} \frac{s^{2}+u^{2}}{t^{2}}
$$

The $u^{2}$-terms arise from scattering of quark currents of different helicities, and spoil the factorisation implied in eq. (2.6) from being exact, necessitating the consideration of kinematic limits of the squared scattering matrix element. This despite the fact that for processes which proceed only through a $t$-channel gluon exchange, the starting expression in terms of spinor strings is already factorised.

In the MRK limit (of infinite rapidity separation between the scattered partons), the expression for the colour and helicity summed and averaged matrix element simplifies to the 2-jet part of eq. (2.6) since all allowed helicity scatterings give the same result in the limit and

$$
|[a b]\langle 21\rangle|=s, \quad t \rightarrow-\left|p_{\perp}\right|^{2} .
$$

This is the lowest order results in eqs. (2.4)-(2.6).

\subsection{Multi-parton production}

In this section we will develop the picture of the scattering of two quark currents to take into account the emission of additional gluons. We first consider adding one extra gluon to the $q Q \rightarrow q Q$ scattering we have taken as our model so far; this may be emitted from the $t$-channel gluon or from each of the external quark lines, figure 7 . 

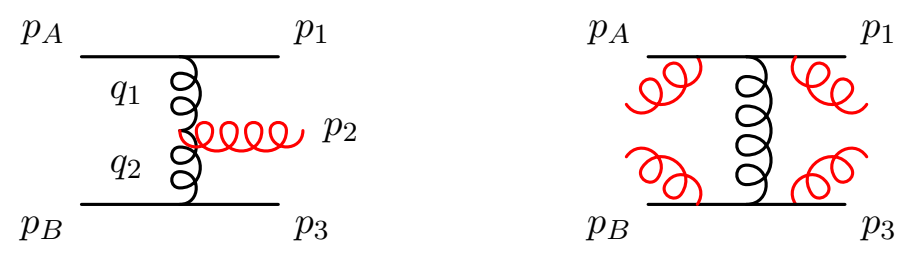

Figure 7. We add contributions where the 3rd jet (red) is emitted from the $t$-channel gluon, and from each of the four external quark lines.

Firstly the three gluon vertex in the $t$-channel emission gives a contribution of

$$
\mathcal{A}_{g}=\frac{-\mathcal{C}_{g} g_{s}^{3}}{t_{1} t_{2}} \bar{u}\left(p_{1}\right) \gamma_{\mu} u\left(p_{A}\right) \bar{u}\left(p_{3}\right) \gamma_{\nu} u\left(p_{B}\right) \varepsilon_{\rho}^{*}\left(\left(q_{1}+q_{2}\right)^{\rho} g^{\mu \nu}+\left(p_{2}-q_{2}\right)^{\mu} g^{\nu \rho}-\left(q_{1}+p_{2}\right)^{\nu} g^{\mu \rho}\right),
$$

where $\mathcal{C}_{g}=T_{a_{1} a_{A}}^{w} f^{w i_{2} v} T_{a_{3} a_{B}}^{v}$. In the MRK limit, we can use eq. (3.1) for the spinor strings, and $q_{1}=p_{3}-p_{B}+p_{2}$ and $q_{2}=p_{a}-p_{1}-p_{2}$ to get

$$
\mathcal{A}_{g} \rightarrow \frac{-2 g_{s}^{3}}{t_{1} t_{2}} \varepsilon_{\rho}^{*}\left(-p_{A}^{\rho}\left(s_{3 B}+2 s_{2 B}\right)+p_{B}^{\rho}\left(s_{1 A}+2 s_{2 A}\right)+\left(q_{1}+q_{2}\right)^{\rho} \hat{s}\right) .
$$

The MRK limit (eq. (2.1)) gives $s_{2 B} \gg s_{3 B}$ and $s_{2 A} \gg s_{1 A}$ so we are left with

$$
\mathcal{A}_{g}^{\mathrm{MRK}}=\frac{-2 g_{s}^{3} \hat{s}}{t_{1} t_{2}} \varepsilon_{\rho}^{*}\left(-2 p_{A}^{\rho} \frac{s_{2 B}}{\hat{s}}+2 p_{B}^{\rho} \frac{s_{2 A}}{\hat{s}}+\left(q_{1}+q_{2}\right)^{\rho}\right) .
$$

We now add the contributions from the emissions of gluons from each external quark line. We treat these emissions as soft, and use Eikonal factors which are valid for a gluon emitted between the quark jets in the MRK limit. These add to give

$$
\mathcal{A}_{q}=\mathcal{A}_{q Q \rightarrow q Q} \times\left(i g_{s}\right) \varepsilon_{\rho}^{*}\left(\mathcal{C}_{1} \frac{p_{1}^{\rho}}{p_{1} \cdot p_{2}}-\mathcal{C}_{A} \frac{p_{A}^{\rho}}{p_{A} \cdot p_{2}}+\mathcal{C}_{3} \frac{p_{3}^{\rho}}{p_{3} \cdot p_{2}}-\mathcal{C}_{B} \frac{p_{B}^{\rho}}{p_{B} \cdot p_{2}}\right) .
$$

The $\mathcal{C}_{i}$ are the relevant colour factors:

$$
\mathcal{C}_{1}=T_{a_{1} b}^{i_{2}} T_{b a_{A}}^{w} T_{a_{3} a_{B}}^{w}, \mathcal{C}_{A}=T_{a_{1} b}^{w} T_{b a_{A}}^{i_{2}} T_{a_{3} a_{B}}^{w}, \mathcal{C}_{3}=T_{a_{1} a_{A}}^{w} T_{a_{3} b}^{i_{2}} T_{b a_{B}}^{w}, \mathcal{C}_{B}=T_{a_{1} a_{A}}^{w} T_{a_{3} b}^{w} T_{b a_{B}}^{i_{2}} .
$$

Now in the MRK limit, $p_{A} \rightarrow p_{1}, p_{B} \rightarrow p_{3}$ and $\mathcal{A}_{q Q \rightarrow q Q}=S_{q Q \rightarrow q Q} / t$, so

$$
\mathcal{A}_{q} \rightarrow \frac{S_{q Q \rightarrow q Q}}{q_{1}^{2} q_{2}^{2}}\left(-i g_{s}^{3}\right) \varepsilon_{\rho}^{*}\left(\left(\mathcal{C}_{1}-\mathcal{C}_{A}\right) q_{1}^{2} \frac{p_{A}^{\rho}}{p_{A} \cdot p_{2}}+\left(\mathcal{C}_{3}-\mathcal{C}_{B}\right) q_{2}^{2} \frac{p_{B}^{\rho}}{p_{B} \cdot p_{2}}\right) .
$$

However, $\left(\mathcal{C}_{1}-\mathcal{C}_{A}\right)=i \mathcal{C}_{g}$ and $\left(\mathcal{C}_{3}-\mathcal{C}_{B}\right)=-i \mathcal{C}_{g}$ so that all five contributions give the same overall colour factor. (The same pairing up is seen when a quark line is replaced by a gluon line, only here it is the Jacobi Identity which can be used to give a single overall factor.)

We could then add eq. (3.14) to eq. (3.11); this gives the form used in [18, 19]. However we choose to first reinstate the full kinematic structure of eq. (3.12) in order to capture more of the original process. We also adapt eq. (3.11) to take account of both $\left\{p_{A}, p_{B}\right\}$ and $\left\{p_{1}, p_{3}\right\}$ to give the following approximation for the $q Q \rightarrow q g Q$-scattering matrix element

$$
\mathcal{A}_{q Q \rightarrow q g Q}=g_{s}^{3} \mathcal{C}_{g} \varepsilon_{\rho}^{*} \frac{S_{q Q \rightarrow q Q}}{q_{1}^{2} q_{2}^{2}} V^{\rho}\left(q_{1}, q_{2}\right)
$$




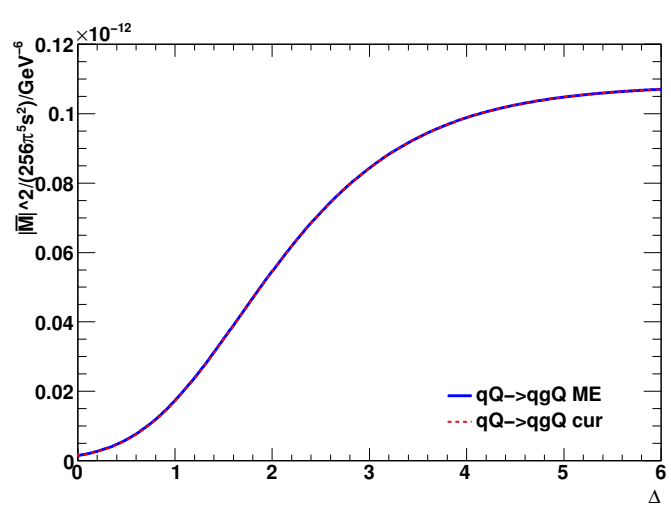

(a)

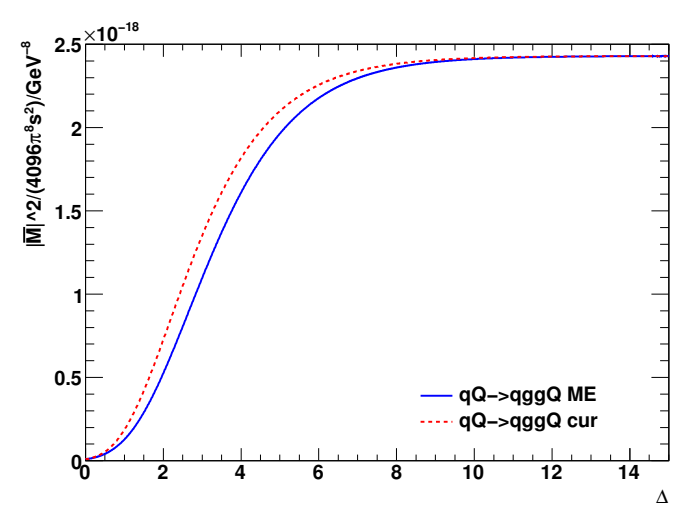

(b)

Figure 8. Results for the matrix elements of (a) $q Q \rightarrow q g Q$ and (b) $q Q \rightarrow q g g Q$ processes respectively. The momentum configurations are as for figure $2(\mathrm{~b})$ and (c) respectively.

where

$$
\begin{aligned}
V^{\rho}\left(q_{1}, q_{2}\right)= & -\left(q_{1}+q_{2}\right)^{\rho} \\
& +\frac{p_{A}^{\rho}}{2}\left(\frac{q_{1}^{2}}{p_{2} \cdot p_{A}}+\frac{p_{2} \cdot p_{B}}{p_{A} \cdot p_{B}}+\frac{p_{2} \cdot p_{3}}{p_{A} \cdot p_{3}}\right)+p_{A} \leftrightarrow p_{1} \\
& -\frac{p_{B}^{\rho}}{2}\left(\frac{q_{2}^{2}}{p_{2} \cdot p_{B}}+\frac{p_{2} \cdot p_{A}}{p_{B} \cdot p_{A}}+\frac{p_{2} \cdot p_{1}}{p_{B} \cdot p_{1}}\right)-p_{B} \leftrightarrow p_{3} .
\end{aligned}
$$

This form of the effective vertex is gauge invariant; the Ward Identity, $p_{g} \cdot V=0$ can easily be checked. The spinor structure is exactly as for $q Q \rightarrow q Q$ and so we find

$$
\begin{aligned}
\left|\overline{\mathcal{M}}_{q Q \rightarrow q g Q}^{t}\right|^{2}= & \frac{1}{4\left(N_{C}^{2}-1\right)}\left\|S_{q Q \rightarrow q Q}\right\|^{2} \\
& \cdot\left(g^{2} C_{F} \frac{1}{t_{1}}\right) \cdot\left(g^{2} C_{F} \frac{1}{t_{2}}\right) \\
& \cdot\left(\frac{-g^{2} C_{A}}{t_{1} t_{2}} V^{\mu}\left(q_{1}, q_{2}\right) V_{\mu}\left(q_{1}, q_{2}\right)\right)
\end{aligned}
$$

The results of this formalism are compared to the full tree-level matrix element for $q Q \rightarrow$ $q g Q$ in figure $8(\mathrm{a})$. The results from the two methods are indistinguishable by eye.

It can be checked that in the MRK limit, eq. (3.16) gives

$$
-V^{\mu}\left(q_{1}, q_{2}\right) V_{\mu}\left(q_{1}, q_{2}\right) \longrightarrow 4 \frac{\left|q_{1 \perp}\right|^{2}\left|q_{2 \perp}\right|^{2}}{\left|p_{2 \perp}\right|^{2}}
$$

The $\left|q_{j \perp}\right|^{2}$ terms in the numerator cancel with the factors of $t_{j}$ in eq. (3.17) to give an MRK limit which agrees with eq. (2.6). Motivated by the structure of eq. (2.6), we describe a 

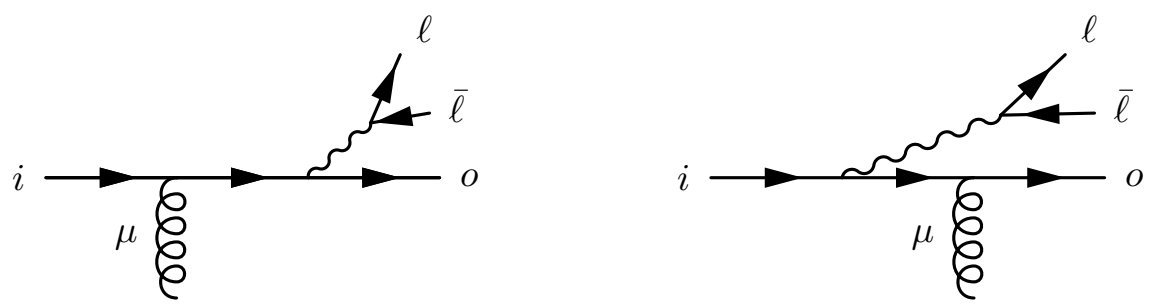

Figure 9. The two diagrams which contribute when a $W$ or $Z$ boson is emitted from a quark current line joined to the rest of the diagram.

general process with $n$ jets in the final state (figure 1) as

$$
\begin{aligned}
\left|\overline{\mathcal{M}}_{q Q \rightarrow q g \ldots g Q}^{t}\right|^{2}= & \frac{1}{4\left(N_{C}^{2}-1\right)}\left\|S_{q Q \rightarrow q Q}\right\|^{2} \\
& \cdot\left(g^{2} C_{F} \frac{1}{t_{1}}\right) \cdot\left(g^{2} C_{F} \frac{1}{t_{n-1}}\right) \\
& \cdot \prod_{i=1}^{n-2}\left(\frac{-g^{2} C_{A}}{t_{i} t_{i+1}} V^{\mu}\left(q_{i}, q_{i+1}\right) V_{\mu}\left(q_{i}, q_{i+1}\right)\right),
\end{aligned}
$$

where $V^{\mu}\left(q_{i}, q_{i+1}\right)$ is the obvious generalisation of eq. (3.16):

$$
\begin{aligned}
V^{\rho}\left(q_{i}, q_{i+1}\right)= & -\left(q_{i}+q_{i+1}\right)^{\rho} \\
& +\frac{p_{A}^{\rho}}{2}\left(\frac{q_{i}^{2}}{p_{i+1} \cdot p_{A}}+\frac{p_{i+1} \cdot p_{B}}{p_{A} \cdot p_{B}}+\frac{p_{i+1} \cdot p_{n}}{p_{A} \cdot p_{n}}\right)+p_{A} \leftrightarrow p_{1} \\
& -\frac{p_{B}^{\rho}}{2}\left(\frac{q_{i+1}^{2}}{p_{i+1} \cdot p_{B}}+\frac{p_{i+1} \cdot p_{A}}{p_{B} \cdot p_{A}}+\frac{p_{i+1} \cdot p_{1}}{p_{A} \cdot p_{1}}\right)-p_{B} \leftrightarrow p_{n} .
\end{aligned}
$$

Each emission vertex comes with a factor of $C_{A}$ in $|\mathcal{M}|^{2}$ because (by the discussion above) there is a $\operatorname{Tr}\left(f^{a b c} f^{a b c}\right)$ in place of a $\delta^{b c} \delta^{b c}$ compared to the process with one fewer jet.

The results for 4 jet final states are shown in figure $8(\mathrm{~b})$ compared to the full matrix element, and show that the formalism performs well. A more detailed study of the results obtained in this formalism for kinematic distributions is presented in section 4.1.

\section{3 $\mathrm{W}$ and $\mathrm{Z}$ boson production in association with jets}

To apply this formalism to $W$ and $Z$ production with jets, we follow the same structure as the pure jets case: modelling both $q Q$ and $q g$ channels on $q Q \rightarrow q(g \ldots g) Q+(V \rightarrow) \ell \bar{\ell}$. When a $W$ or $Z$ is emitted from a quark line, the two diagrams shown in figure 9 need to be considered. The gluon line with free index $\mu$ links this current to the rest of the diagram. The two diagrams add to give

$$
J_{V}^{\mu}(i, \ell, \bar{\ell},, o)=\left(\frac{\bar{u}_{o} \gamma^{\alpha}\left(\not p_{o}+\not \ell_{\ell}+\not \bar{\ell}_{\bar{\ell}}\right) \gamma^{\mu} u_{i}}{t_{o \ell \bar{\ell}}}+\frac{\bar{u}_{o} \gamma^{\mu}\left(\not p_{i}-\not p_{\ell}-\not \bar{\ell}_{\bar{\ell}}\right) \gamma^{\alpha} u_{i}}{t_{i \ell \bar{\ell}}}\right) \bar{u}_{\ell} \gamma_{\alpha} v_{\bar{\ell}}
$$


We can rewrite this in terms of manageable $\overline{\text { spinor}}$-matrix-spinor pieces as:

$$
\begin{aligned}
J_{V}^{\mu}(i, \ell, \bar{\ell},, o)=\left(\frac{2 p_{o}^{\alpha}\left[\bar{u}_{o} \gamma^{\mu} P_{i} u_{i}\right]+\left[\bar{u}_{o} \gamma^{\alpha} P_{o} u_{\bar{\ell}}\right]\left[\bar{u}_{\bar{\ell}} \gamma^{\mu} P_{i} u_{i}\right]+\left[\bar{u}_{o} \gamma^{\alpha} P_{o} u_{\ell}\right]\left[\bar{u}_{\ell} \gamma^{\mu} P_{i} u_{i}\right]}{t_{o \ell \bar{\ell}}}\right. & \\
+ & \left.\frac{2 p_{i}^{\alpha}\left[\bar{u}_{o} \gamma^{\mu} P_{o} u_{i}\right]-\left[\bar{u}_{o} \gamma^{\mu} P_{o} u_{\ell}\right]\left[\bar{u}_{\ell} \gamma^{\alpha} P_{i} u_{i}\right]-\left[\bar{u}_{o} \gamma^{\mu} P_{o} u_{\bar{\ell}}\right]\left[\bar{u}_{\bar{\ell}} \gamma^{\alpha} P_{i} u_{i}\right]}{t_{i \ell \bar{\ell}}}\right) \\
& \times\left[\bar{u}_{\ell} \gamma_{\alpha} P_{\ell} u_{\bar{\ell}}\right],
\end{aligned}
$$

where $P_{x}$ is the projection operator $P_{ \pm}=\left(1 \pm \gamma_{5}\right) / 2$ according to the helicity of particle $x$. The current is zero unless $P_{o}=P_{i}$.

There are two classes of subprocess to consider, those where

1. the $W$ or $Z$ boson can only be emitted from one quark line, which is the case in all $q g$ channels and certain $q Q$ channels for $W$ production, or

2. the $W$ or $Z$ boson can be emitted from either quark line.

In the following, without loss of generality, we pick a specific example subprocess for each case: for case $1, u d \rightarrow d d(W \rightarrow) \nu_{\ell} \bar{\ell}$ and for case $2, u d \rightarrow u d(Z \rightarrow) \bar{\ell}$.

Case 1 is straightforward and the analogue of equation (3.4) is

$$
S_{u d \rightarrow d d \nu_{\ell} \bar{\ell}}^{-h_{b} \rightarrow-h_{2}--}=J_{V \mu}(i, \ell, \bar{\ell}, o) g^{\mu \nu}\left\langle 2 h_{2}|\nu| b h_{b}\right\rangle .
$$

Again we use double vertical line notation to symbolise the sum over helicities of each $|S|^{2}$. We write

$$
\begin{aligned}
\left.|| S_{u d \rightarrow d \nu_{\ell} \bar{\ell} d}\right|^{2}= & \frac{g_{W}^{4}}{4} \sum_{h_{b}, h_{2}}\left|S_{u d \rightarrow d \nu_{\ell} \bar{\ell} d}^{-h_{b} \rightarrow-h_{2}--}\right|^{2}, \\
\left|\overline{\mathcal{M}}_{u d \rightarrow d \nu_{\ell} \bar{\ell} d}^{t}\right|^{2}= & \frac{1}{4\left(N_{C}^{2}-1\right)}\left\|S_{u d \rightarrow d \nu_{\ell} \ell d}\right\|^{2} \\
& \cdot\left(g^{2} C_{F} \frac{1}{t_{1}}\right) \\
& \cdot\left(g^{2} C_{F} \frac{1}{t_{2}}\right)
\end{aligned}
$$

where now $t_{1}=\left(p_{a}-p_{1}-p_{\nu}-p_{\ell}\right)^{2}$ and $t_{2}=\left(-p_{b}+p_{2}\right)^{2}$ (still $t_{1}=t_{2}$ in this $2 j$ case).

In case 2 , it is similar but slightly more complicated as one must divide by $t$ s before squaring so we get

$$
\begin{aligned}
\left|\overline{\mathcal{M}}_{u d \rightarrow u \ell \bar{\ell} d}^{t}\right|^{2}= & \frac{1}{4\left(N_{C}^{2}-1\right)} \cdot\left(g^{2} C_{F}\right) \cdot\left(g^{2} C_{F}\right) \\
& \times \sum_{\substack{h_{a}, h_{b}, h_{1}, h_{2}, h_{\ell}, h_{\bar{\ell}}}} k_{Z \ell \bar{\ell}}^{2} \mid k_{u} J_{V \mu}(a, \ell, \bar{\ell}, 1) g^{\mu \nu}\left\langle 2 h_{2}|\nu| b h_{b}\right\rangle \cdot \frac{1}{t_{t}} \\
& \\
& +\left.k_{d}\left\langle 1 h_{1}|\nu| a h_{a}\right\rangle g^{\mu \nu} J_{V \nu}(b, \ell, \bar{\ell}, 2) \cdot \frac{1}{t_{b}}\right|^{2}
\end{aligned}
$$




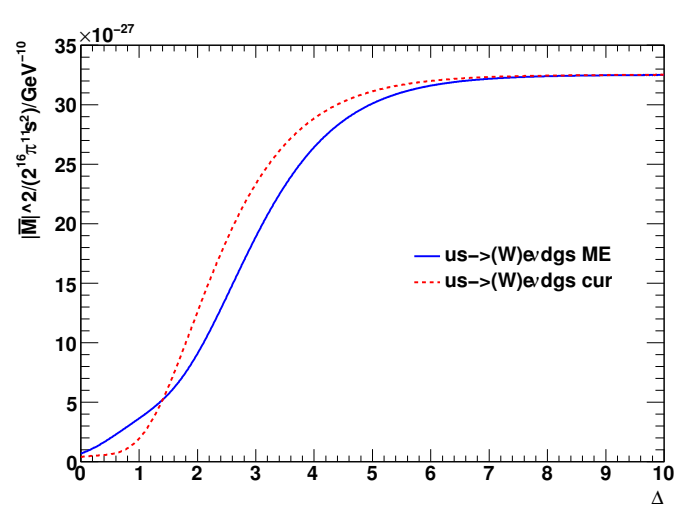

(a)

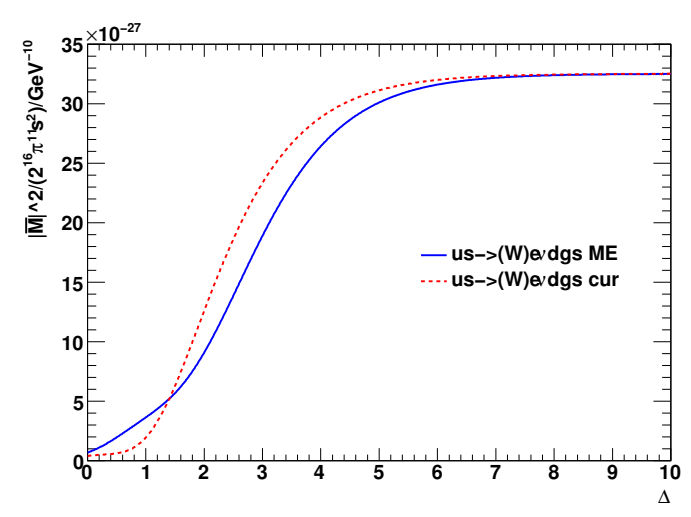

(b)

Figure 10. A comparison of the matrix elements obtained from Madgraph and from our formalism for (a) $W+3 j$ and (b) $W+4 j$. The jets have rapidities $\Delta, 0,-\Delta$ in (a), and $\Delta, \Delta / 3,-\Delta / 3,-\Delta$ in (b). The full momentum configurations are in appendix B.

where $k_{u, d}$ are the different couplings of top and bottom emission, $k_{Z \ell \bar{\ell}}$ is the $Z$ coupling to the leptons, $t_{t}=\left(p_{a}-p_{1}-p_{\ell}-p_{\bar{\ell}}\right)^{2}$ and $t_{b}=\left(p_{b}-p_{2}-p_{\ell}-p_{\bar{\ell}}\right)^{2}$.

As in the case of pure jets, these results are exact for $q Q \rightarrow q Q+\ell \bar{\ell}$. We now want to extend this to $2 j \rightarrow n j+\ell \bar{\ell}$ for $n>2$. In case 1 , the inclusion of further gluon emissions (ordered in rapidity) is done in exactly the same way as for the pure jet case, outlined in section 3.2. The results are shown in figure 10 for $W+3 j$ and $W+4 j$.

Case 2 is very similar, except the Lipatov vertices must be included in the analogue of equation (3.26) before squaring. For example, consider our sample $u d$ process with $n$ jets in the final state, figure 11. To make the (simple) structure clear, we introduce the shorthands

$$
a_{t}=J_{V \mu}(a, \ell, \bar{\ell}, 1) g^{\mu \nu}\left\langle 2 h_{2}|\nu| b h_{b}\right\rangle \quad \text { and } \quad a_{b}=\left\langle 1 h_{1}|\nu| a h_{a}\right\rangle g^{\mu \nu} J_{V \nu}(b, \ell, \bar{\ell}, 2)
$$

for the current parts. Then the $|\ldots|^{2}$ expression in equation (3.26) becomes

$$
\begin{gathered}
\left(k_{u} a_{t} \cdot \frac{V^{\rho_{1}}\left(q_{t_{1}}, q_{t_{2}}\right) \ldots V^{\rho_{n-2}}\left(q_{t_{n-2}}, q_{t_{n-1}}\right)}{t_{t_{1}} \ldots t_{t_{n-1}}}+k_{d} a_{b} \cdot \frac{V^{\rho_{1}}\left(q_{b_{1}}, q_{b_{2}}\right) \ldots V^{\rho_{n-2}}}{t_{b_{1}} \ldots t_{b_{n-1}}}\right) \\
\quad \times\left(-g_{\rho_{1} \sigma_{1}}\right) \ldots\left(-g_{\rho_{n-2} \sigma_{n-2}}\right) \\
\quad \times\left(k_{u} a_{t}^{*} \cdot \frac{V^{\sigma_{1}}\left(q_{t_{1}}, q_{t_{2}}\right) \ldots V^{\sigma_{n-2}}\left(q_{t_{n-2}}, q_{t_{n-1}}\right)}{t_{t_{1}} \ldots t_{t_{n-1}}}+k_{d} a_{b}^{*} \cdot \frac{V^{\sigma_{1}}\left(q_{b_{1}}, q_{b_{2}}\right) \ldots V^{\sigma_{n-2}}}{t_{b_{1}} \ldots t_{b_{n-1}}}\right) \\
=(-1)^{n}\left(k_{u}^{2}\left|a_{t}\right|^{2} \frac{V\left(q_{t_{1}}, q_{t_{2}}\right)^{2} \ldots V\left(q_{t_{n-2}}, q_{t_{n-1}}\right)^{2}}{t_{t_{1}}^{2} \ldots t_{t_{n-1}}^{2}}+k_{d}^{2}\left|a_{b}\right|^{2} \frac{V\left(q_{b_{1}}, q_{b_{2}}\right)^{2} \ldots V\left(q_{b_{n-2}}, q_{b_{n-1}}\right)^{2}}{t_{b_{1}}^{2} \ldots t_{b_{n-1}}^{2}}\right. \\
\left.\quad+2 k_{u} k_{d}\left(a_{t} a_{b}^{*}+a_{t}^{*} a_{b}\right) \frac{V\left(q_{t_{1}}, q_{t_{2}}\right) \cdot V\left(q_{b_{1}}, q_{b_{2}}\right) \ldots V\left(q_{t_{n-2}}, q_{t_{n-1}}\right) \cdot V\left(q_{b_{n-2}}, q_{b_{n-1}}\right)}{\left.t_{t_{1}} \ldots t_{t_{n-1}}\right)}\right),
\end{gathered}
$$




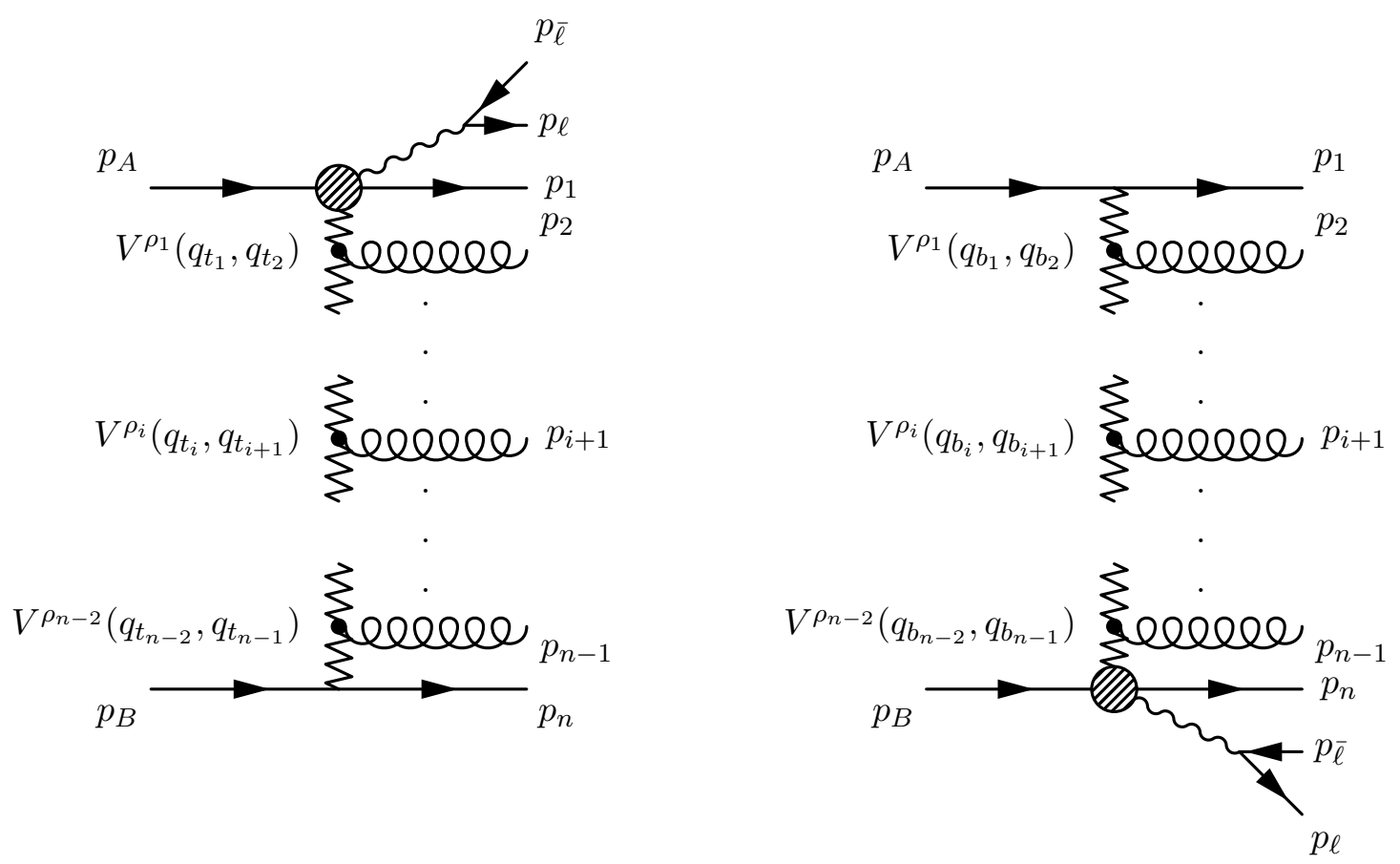

Figure 11. The two diagrams which are added at amplitude level for a Case 2 process with an $n$ jet final state.

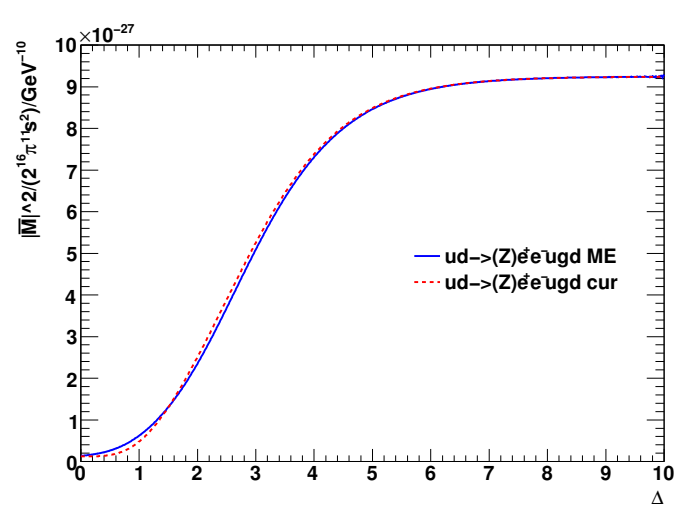

(a)



(b)

Figure 12. A comparison of the matrix elements obtained from Madgraph and from our formalism for (a) $Z+3 j$ and (b) $Z+4 j$ (Case 2). The rapidities are as in figure 10. The full momentum configurations are in appendix $\mathrm{B}$.

where $t_{t i}$ and $t_{b i}$ are the analogues of equation (2.3) for top and bottom line emission respectively. What is particularly appealing about this form is that there are only ever 3 terms, independent of the number of final jets. The results are shown in figure 12 for $Z+3 j$ and $Z+4 j$.

\subsection{Higgs boson production in association with jets}

In this study we will consider just the production of a Higgs boson in-between (in rapidity) jets, since this will nicely illustrate the increased predictive power of the factorised current 
formalism over the formalism based on kinematic limits of amplitudes. The production of a Higgs boson "outside" jets could be fully included by obtaining results for "Higgs+gluon"impact factors (see ref. [15] for details) also within the current formalism.

Let us start by considering the scattering $q^{-} Q^{-} \rightarrow q^{-} Q^{-} H$ with the scattering matrix element

$$
M_{q^{-} Q^{-} \rightarrow q^{-} Q^{-} H}=\langle 1|\mu| a\rangle \frac{g^{\mu \sigma_{1}}}{q_{1}^{2}} V_{\sigma_{1} \sigma_{2}}^{H}\left(q_{1}, q_{2}\right) \frac{g^{\sigma_{2} \nu}}{q_{2}^{2}}\langle 2|\nu| b\rangle,
$$

where the effective coupling of two off-shell gluons to the Higgs boson field through a topquark triangle is described by the vertex obtained in the infinite top-mass limit (please note however that the picture of factorised amplitudes does not rely on taking the infinite top-mass limit)

$$
V_{H}^{\sigma_{1} \sigma_{2}}\left(q_{1}, q_{2}\right)=g^{\sigma_{1} \sigma_{2}} q_{1} \cdot q_{2}-q_{1}^{\sigma_{2}} q_{2}^{\sigma_{1}}
$$

Here, $q_{1}=p_{a}-p_{1}, q_{2}=q_{1}-p_{H}$. Again, we will use the sum of all allowed helicity configurations as the model for all partonic channels. Let us define the spinor/Lorentz string

$$
S_{q Q \rightarrow q H Q}^{h_{a} h_{b} \rightarrow h_{1} h_{2}}\left(q_{1}, q_{2}\right)=\left\langle 1 h_{1}|\mu| a h_{a}\right\rangle g^{\mu \sigma_{1}} V_{\sigma_{1} \sigma_{2}}^{H}\left(q_{1}, q_{2}\right) g^{\sigma_{2} \nu}\left\langle 2 h_{2}|\nu| b h_{b}\right\rangle .
$$

Setting again

$$
\left\|S_{q Q \rightarrow q H Q}\left(q_{1}, q_{2}\right)\right\|^{2}=\sum_{h_{a}, h_{a}, h_{b}, h_{2}}\left|S_{q Q \rightarrow q H Q}^{h_{a} h_{b} \rightarrow h_{1} h_{2}}\left(q_{1}, q_{2}\right)\right|^{2}
$$

the helicity and colour summed and averaged square of the matrix element is given by

$$
\begin{aligned}
\left|\overline{\mathcal{M}}_{q Q \rightarrow q H Q}^{t}\right|^{2}= & \frac{1}{4\left(N_{C}^{2}-1\right)}\left\|S_{q Q \rightarrow q H Q}\left(q_{1}, q_{2}\right)\right\|^{2} \\
& \cdot\left(g^{2} C_{F} \frac{1}{t_{1}}\right) \\
& \cdot\left(\frac{1}{t_{1}}\left(\frac{\alpha_{s}}{6 \pi v}\right)^{2} \frac{1}{t_{2}}\right) \\
& \cdot\left(g^{2} C_{F} \frac{1}{t_{2}}\right) .
\end{aligned}
$$

Compared to the $2 \rightarrow 2$ current scattering of eq. (3.4), an extra Lorentz-tensor has been inserted in the spinor/Lorentz structure, contracted with the external currents (eq. (3.31)). In the square of the amplitude, an extra inverse power of the square of the momentum either side of the Higgs boson vertex has appeared. This is the same situation as with the emission of a gluon by use of the effective vertex eq. (3.16). Therefore, the approximation for Higgs production in association with three jets will be based on the physical picture of (high energy) current-current scattering with rapidity ordered $t$-channel couplings of the effective gluon emission vertex (eq. (3.16)) and the $g^{*} g^{*} H$-vertex of eq. (3.30), in line with the four-jet formalism. Therefore, for the multi-gluon emissions, we will take $q_{1}, q_{2}$ in the 


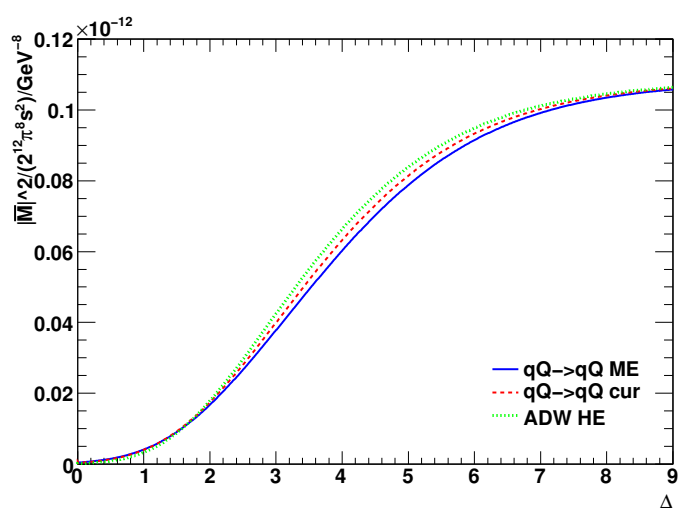

Figure 13. The full matrix element for $q Q \rightarrow q H g Q$, the result of eq. (3.34) ("cur") and the approximation of $[18,19]$ ( "ADW HE").

argument of the Higgs boson vertex to be the $t$-channel momentum of the propagators either side of the Higgs boson-vertex.

The approximation for the colour and helicity summed and averaged square of the matrix element for the rapidity ordering implied by the subscript

$$
\begin{aligned}
\left|\overline{\mathcal{M}}_{q Q \rightarrow q H g Q}^{t}\right|^{2}= & \frac{1}{4\left(N_{C}^{2}-1\right)}\left\|S_{q Q \rightarrow q H Q}\left(q_{1}, q_{2}\right)\right\|^{2} \\
& \cdot\left(g^{2} C_{F} \frac{1}{t_{1}}\right) \\
& \cdot\left(\frac{1}{t_{1}}\left(\frac{\alpha_{s}}{6 \pi v}\right)^{2} \frac{1}{t_{2}}\right) \\
& \cdot\left(g^{2} C_{A} \frac{1}{t_{2}}(-V . V) \frac{1}{t_{3}}\right) \\
& \cdot\left(g^{2} C_{F} \frac{1}{t_{3}}\right),
\end{aligned}
$$

where $V^{\mu}=V^{\mu}\left(q_{2}, q_{3}\right)$ of eq. (3.16), $q_{1}=p_{a}-p_{1}, q_{2}=q_{1}-p_{H}, q_{3}=q_{2}-p_{2}$, and $t_{i}=q_{i}^{2}$, with the momentum of the quark currents $\left(p_{a}, p_{1}\right)$ and $\left(p_{b}, p_{3}\right)$ respectively. Once again, the factorised formalism for the scattering is extremely simple, and the generalisation to multiple emissions is straightforward. Again, the approximations for the gluon channels differ only by colour factors (multiplication by $C_{A} / C_{F}$ for each parton line replaced).

We show in figure 13 the results of this formalism compared to the full matrix element. We also show the approximation of ref. $[18,19]$. We will in section 4.4 investigate the degree to which this formalism captures the physics of the full scattering amplitude.

\subsection{Virtual corrections and regularisation}

So far, we have discussed how the real emission is approximated to all orders. In this subsection we will discuss how the MRK limit of the virtual corrections can be implemented according to the Lipatov Ansatz. This will facilitate the construction of a regularised, allorder scattering matrix element for each $n$-parton exclusive final state. We emphasise that: 
a) in the current study, we construct scattering matrix elements only for rapidity orderings which allow colour octet-exchanges between each pair of rapidity ordered particles, b) the contribution from other rapidity orderings are systematically suppressed by powers of invariant masses; some of these configurations would arise when considering the next-toleading contributions to the factorisation, but can still be included in a $t$-channel effective description [17]. The degree to which the leading flavour assignments dominate the $n$-jet cross sections was discussed in e.g. ref. $[18,19]$ and are repeated in section 4.4 . We leave the treatment of the sub-leading configurations to a later study.

The Lipatov Ansatz states that the order by order, the virtual corrections to the full $n$-parton scattering amplitude in the MRK limit can be obtained by replacing in the scattering amplitudes

$$
\frac{1}{t_{i}} \rightarrow \frac{1}{t_{i}} \exp \left[\hat{\alpha}\left(q_{i}\right)\left(y_{i-1}-y_{i}\right)\right]
$$

with

$$
\begin{aligned}
\hat{\alpha}\left(q_{i}\right) & =\alpha_{s} C_{A} t_{i} \int \frac{d^{2} k_{\perp}}{(2 \pi)^{2}} \frac{1}{k_{\perp}^{2}\left(q_{i}-k\right)_{\perp}^{2}} \\
& \rightarrow-g^{2} C_{A} \frac{\Gamma(1-\varepsilon)}{(4 \pi)^{2+\varepsilon}} \frac{2}{\varepsilon}\left(\mathbf{q}^{2} / \mu^{2}\right)^{\varepsilon}
\end{aligned}
$$

This ansatz for the exponentiation of the virtual corrections in the appropriate limit of the $n$-parton scattering amplitude has been proved to even the sub-leading level [16, 17, 21, 22].

Our discussion of the regularisation of the soft divergences will follow closely the discussion in ref. [19]. We will show that order by order in $\alpha_{s}$, the soft divergence for the emission of gluons cancels with the soft divergence from the virtual corrections implemented according to the Lipatov Ansatz for the resummed $t$-channel propagator. Consider the limit where the transverse momentum of the $i$ 'th emitted gluon is vanishing. In this limit,

$$
\left|\overline{\mathcal{M}}_{p_{a} p_{b} \rightarrow p_{1} \cdots p_{i-1} p_{i} p_{i+1} \cdots p_{n}}^{t}\right|^{2} \stackrel{\mathbf{p}_{i}{ }^{2} \rightarrow 0}{\longrightarrow}\left(\frac{4 g^{2} C_{A}}{\mathbf{p}_{i}{ }^{2}}\right)\left|\overline{\mathcal{M}}_{p_{a} p_{b} \rightarrow p_{1} \cdots p_{i-1} p_{i+1} \cdots p_{n}}^{t}\right|^{2},
$$

where the matrix element on the r.h.s. has $n-1$ final state particles, and $\mathbf{p}_{i}^{2}$ is the sum of the squares of the transverse components of $p_{i}$ in the Euclidean metric. By integrating over the soft region $\mathbf{p}_{i}^{2}<\lambda^{2}$ of phase space in $D=4+2 \varepsilon$ dimensions we find

$$
\begin{aligned}
\int_{0}^{\lambda} & \frac{\mathrm{d}^{2+2 \varepsilon} \mathbf{p} \mathrm{d} y_{i}}{(2 \pi)^{2+2 \varepsilon} 4 \pi}\left(\frac{4 g^{2} C_{A}}{\mathbf{p}^{2}}\right) \mu^{-2 \varepsilon} \\
& =\frac{4 g^{2} C_{A}}{(2 \pi)^{2+2 \varepsilon} 4 \pi} \Delta y_{i-1, i+1} \frac{\pi^{1+\varepsilon}}{\Gamma(1+\varepsilon)} \frac{1}{\varepsilon}\left(\lambda^{2} / \mu^{2}\right)^{\varepsilon} .
\end{aligned}
$$

The square of the matrix element on the left hand side of eq. (3.38) contains the exponential $\exp \left(2 \alpha\left(q_{i}\right) \Delta y_{i-1, i+1}\right)$. By expanding the exponential to first order in $\alpha_{s}$ and in $\varepsilon$, the resulting pole in $\varepsilon$ does indeed cancel that of eq. (3.39), and the combined effect 
of one soft real emission and the first term in the expansion of the Reggeised propagator is a factor

$$
\Delta y_{i-1, i+1} \frac{\alpha_{s} N_{C}}{\pi} \ln \left(\frac{\lambda^{2}}{\mathbf{q}^{2}}\right)
$$

multiplying the $(n-1)$-particle matrix element.

It is clear that the nested rapidity integrals of additional soft radiation in the $t$-channel factorised multi-parton amplitudes will build up the exponential needed to cancel the poles from the virtual corrections to all orders in $\alpha_{s}$. The divergence arising from a given real emission is therefore cancelled by that arising from the virtual corrections in the Reggeised $t$-channel propagator of the matrix element without the relevant real emission. Furthermore, the organisation of the cancellation of infra-red poles can be achieved with a simple phase-space slicing. Since the $t$-channel factorised matrix elements are very fast to evaluate and the regularisation procedure does not add any complexity (because of the simple IR structure of the $t$-channel factorised matrix elements), the radiative corrections to all orders can be constructed as an explicit phase space integral over each number of resolved $\left(\left|\mathbf{k}_{i}\right|^{2}>\lambda^{2}, i=2, \ldots n-1\right)$ gluons emitted. The cancellation of the poles in $\varepsilon$ ensures that the logarithmic dependence on $\lambda$ generated by the lower limit on the transverse momentum integrals cancels with the logarithmic $\lambda$-dependence of the virtual + unresolvedreal correction. This is similar to the explicit construction of the solution to the BFKL evolution, where the very weak dependence of the solution on $\lambda$ at leading logarithmic accuracy was studied in ref. [23, 24], and in ref. [25] at next-to-leading logarithmic accuracy.

The construction of an explicit integration over emissions to all orders relies on an efficient phase-space generator $[18,19]$, which should sample final states with the number of particles varying by more than an order of magnitude. The problem is significantly different to that of a so-called general purpose Monte Carlo (Pythia [1], Herwig [2], SHERPA [3]) where also the number of final state particles varies, since in these the approximation to the virtual corrections is defined such that the emission of particles is unitary, i.e. does not change the total cross section, which allows for a simple probabilistic interpretation. In the problem at hand, (an approximation to) the virtual corrections are also calculated, and introduce a suppression of the regularised matrix element for any final state with a finite number of partons as the rapidity length of the event is increased. This is countered by the (positive) contribution from the emission of additional gluons, and introduces a correlation between the number of final state partons and the typical rapidity length of an event, which depends also on the transverse momenta. This probabilistic correlation is absolutely crucial to incorporate in the phase space generator in order to obtain numerical stability in finite amount of time. Such a phase space integrator can be efficiently implemented by following the ideas of ref. [26].

\section{Applications}

The framework developed here is only useful if it is relevant to the current generation of colliders. In this section, we compare the results of using the $t$-channel factorised matrix 
elements order by order with those obtained with full tree-level matrix elements (extracted from MadGraph [13]) for a $10 \mathrm{TeV}$ proton-proton-collider. We will compare both rapidity and transverse momentum distributions for a representative set of processes with a minimal set of cuts. Since the only difference between the two calculations presented is the evaluation of the hard scattering matrix element, the choice of coupling or parton distribution functions is largely irrelevant for the purpose of comparison and judging the accuracy of the simple picture for higher order corrections. In the later construction of the resummation, we envisage using a NLO set of pdfs. Therefore, we choose also in this study to apply the MSTW2008NLO set [27] of pdfs, so that one can judge already now the level of corrections which will arise from matching the resummation to fixed order accuracy. We will use the $k_{t}$-algorithm as implemented in ref. [28] to define jets.

In the following, we will only integrate over final states in which the partons are arranged in rapidity such that colour octets can be exchanged between all rapidity-neighbours; this constraint is relevant only for some partonic channels. Other contributions are systematically suppressed by the invariant mass between the non-ordered partons, and the relevance of this suppression was checked in an earlier publication [19]. We will repeat this analysis for the updated set of cuts in section 4.4. The non-leading contributions can also be included in a $t$-channel factorised formalism [17], but we have not yet done so, and so we will compare with the results from full tree-level QCD only of the final states which we claim to reproduce.

We emphasise that the following results will form the basis of a resummation procedure, in which the discrepancies between the full tree-level results and those obtained using the approximation of a $t$-channel factorised scattering can be corrected for order-by-order, where the full result is known. However, the close resemblance between the results obtained order-by-order in the simple picture (allowing all-order constructions) and with full QCD should instill trust in the results which will be obtained from the resummation procedure.

\subsection{Pure jets}

Since the two-jet cross section is reproduced exactly for the pure quark channels, we will present comparisons only for the three and four-jet channels. We will use the following minimal set of cuts

$$
p_{j_{\perp}}>40 \mathrm{GeV} \quad\left|y_{j}\right|<4.5
$$

In figure 14 we compare the differential cross section with respect to the rapidity difference and the azimuthal angle between the most forward and backward jet between the full leading order matrix element and the approximation of this obtained in the $t$-channel factorised approach. In the case of $u d$-scattering $(a)-(b)$, we note that because of the valence quark contribution, the rapidity-distribution is peaked at rapidities around 4 , and a very good agreement between the result obtained within the full tree-level and the simpler $t$-channel factorised approach; we emphasise that no constraints on the minimal separation between jets have been applied. Neither a constraint on the similarity of transverse scales in accordance with eq. (2.1). 
In the $q Q$-channel, the $t$-channel factorised picture obviously works particularly well across all rapidities. Also the azimuthal distribution is described very well. Indeed, the (small) discrepancy is isolated to the collinear region, where the forward and backward jet is back-to-back in azimuth, and thus the third hard jet is radiated in the same direction as one of the other jets. A discrepancy in this region is hardly surprising, since the collinear singularity is not included in our $t$-channel factorised description. For the $g q$ and $g g$ channel, the peak in the rapidity distribution obtained with the full LO matrix element is at ever decreasing rapidities; slightly above 2 for the channel $g g \rightarrow g g g$. As expected, in these channels the $t$-channel dominance is only reached for larger rapidities as compared to the $u d$-channels. However, we emphasise that matching corrections can be taken into account in the resummation procedure which will be built upon these approximations, such that for smaller rapidities, where the first few orders of the perturbative expansion is sufficient (i.e. the effects of the resummation are small), full LO results will be used, and for larger rapidities where the resummation effects are important, the approximations fare better and so the approximations can be better trusted. This is achieved following the matching procedure discussed in ref. [19].

In figure 15 we have plotted the similar distributions in the 4 -jet cases. We emphasise that these (and those in the subsequent sections) are not normalised distributions - the total rates and distributions are really approximated by the simple $t$-channel factorised framework as well as indicated in the figures. We note that for all the channels, the peak in the rapidity distributions have moved to slightly larger rapidities (roughly one unit) than in the equivalent three-jet channels. This is simply because of two effects: 1) the opening of phase space and 2) that the partonic cross section reaches the asymptotic (and large) values at increasing rapidity spans for an increasing number of final state particles (see figure 2). We also note that the importance of the $q g$ channel compared with the $g g$-one increases with the jet count.

\section{2 $\mathrm{W}+$ jets}

We now compare the results for the production of a (leptonically decaying) $W$ boson in association with three and four jets. For brevity, we only show plots for the dominant $u g \rightarrow$ $e^{+} \nu_{e} d g(g) g g$ channel; the $t$-channel factorised matrix elements reproduce the channels with an initial state of only quarks even better.

We apply the following set of cuts

$$
\begin{array}{ll}
p_{j_{\perp}}>40 \mathrm{GeV} & p_{e \perp}>20 \mathrm{GeV} \\
\left|y_{j}\right|<4.5 & \left|y_{e}\right|<2.5 \\
& p_{\nu \perp}>20 \mathrm{GeV}
\end{array}
$$

The tree-level description of the channels $q Q \rightarrow q^{\prime} Q(W \rightarrow)$ e $\nu$ are reproduced exactly; this is an improvement over the earlier approximations of ref. [14], where kinematical limits of also the $W$ (and its decay products) had to be applied in order to extract the relevant impact factor. In particular, the $W$ had to be assumed to be produced in the forward region, with its rapidity increasing with that of the emitting quark. This assumption is in contradiction with the requirement of the charged lepton of the $W$ decay products to 


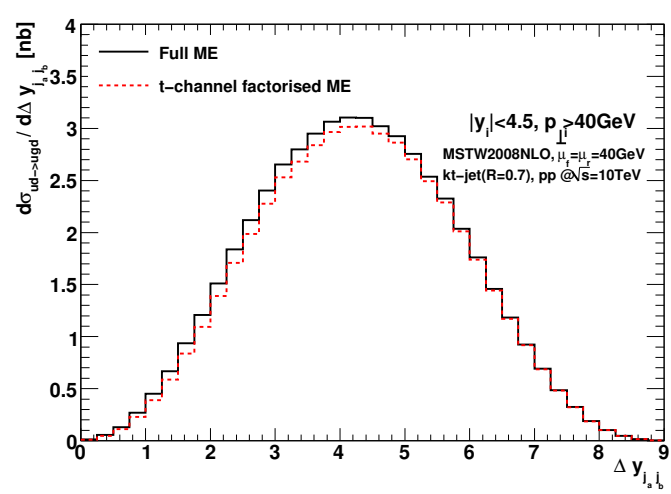

(a)

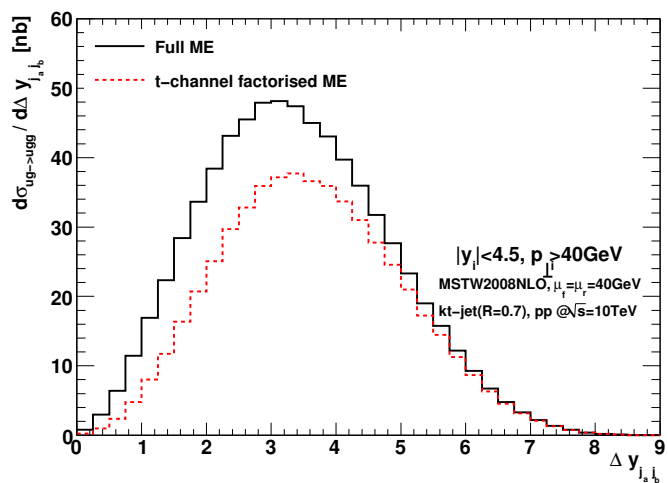

(c)

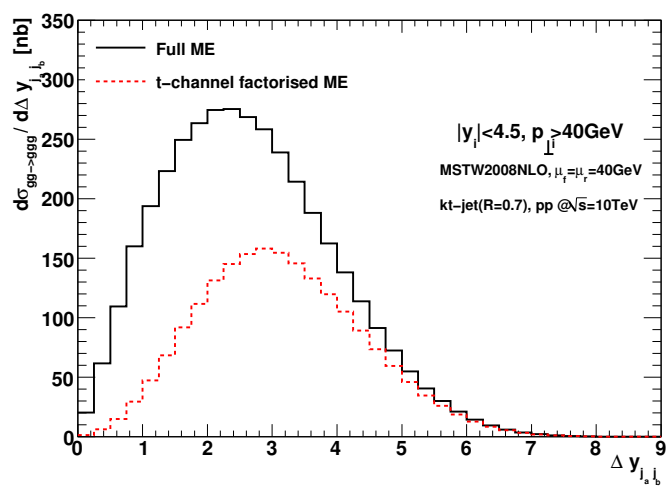

(e)

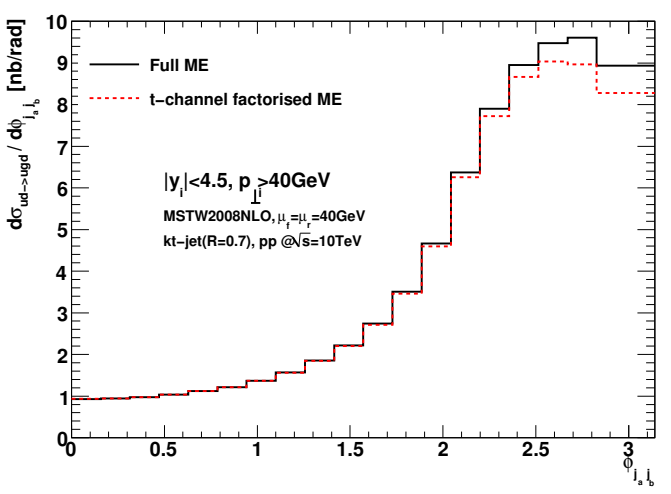

(b)

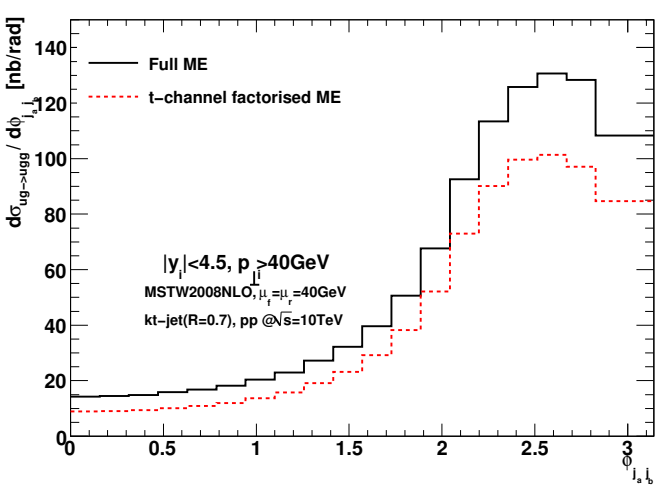

(d)

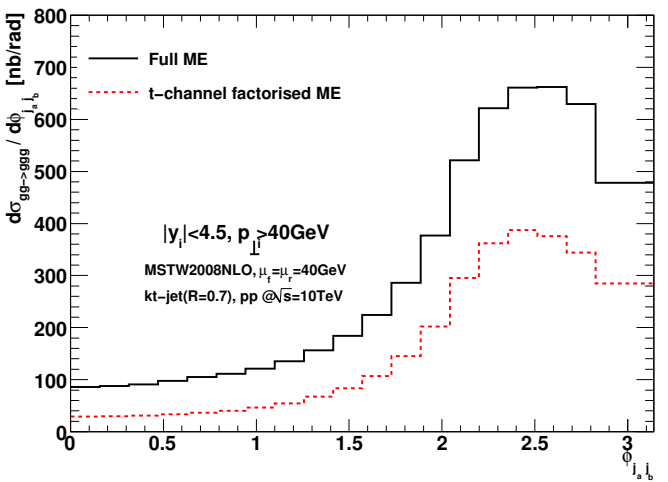

(f)

Figure 14. A comparison between the differential 3-jet cross section obtained using lowest order matrix elements obtained from Madgraph and the $t$-channel factorised formalism for the process (a)-(b) $u d \rightarrow u g d$ with the $u$-quark incoming in the positive $z$-direction. (a) the rapidity difference between the most forward and backward jet (b) the distribution on the azimuthal angle between the most forward and backward jet. Similarly for $u g \rightarrow u g g$ (c)-(d) and $g g \rightarrow g g g$ (e)-(f).

be central for detection. The formalism developed in section 3.3 requires no kinematical constraints to be placed on the $W$ or its decay products in order to extract the building 


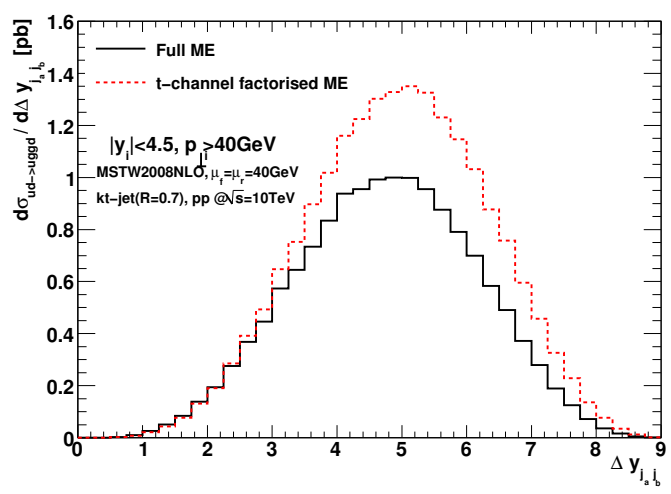

(a)

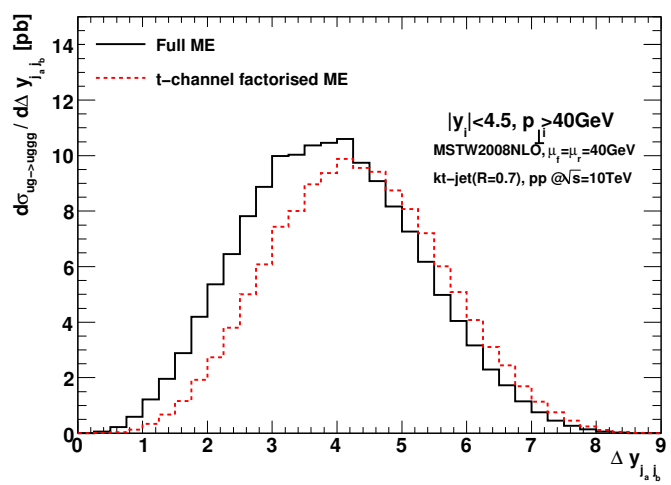

(c)

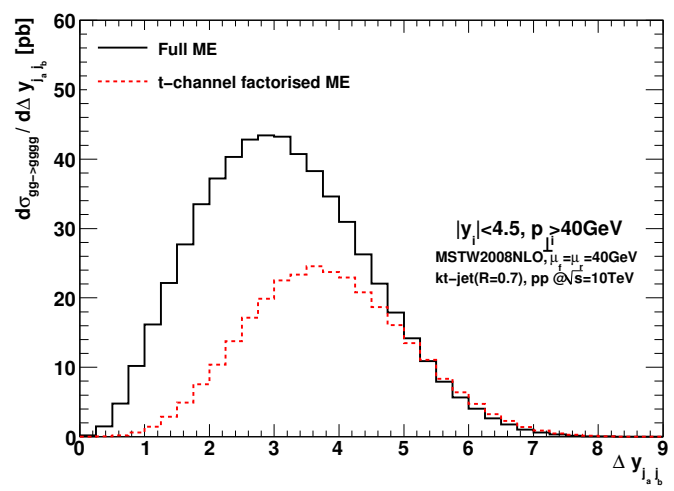

(e)

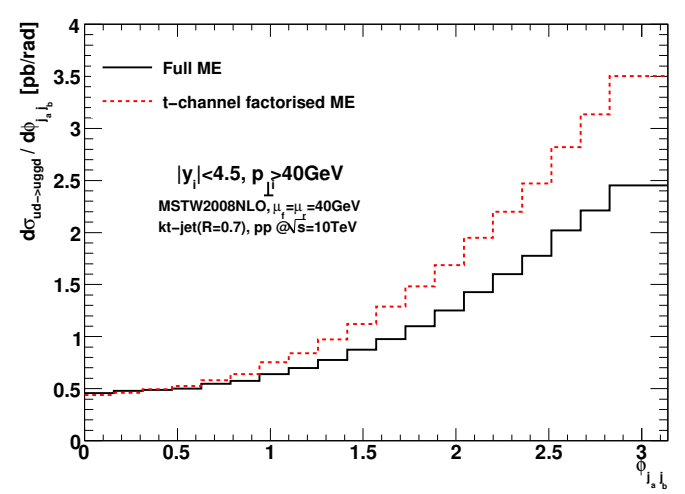

(b)

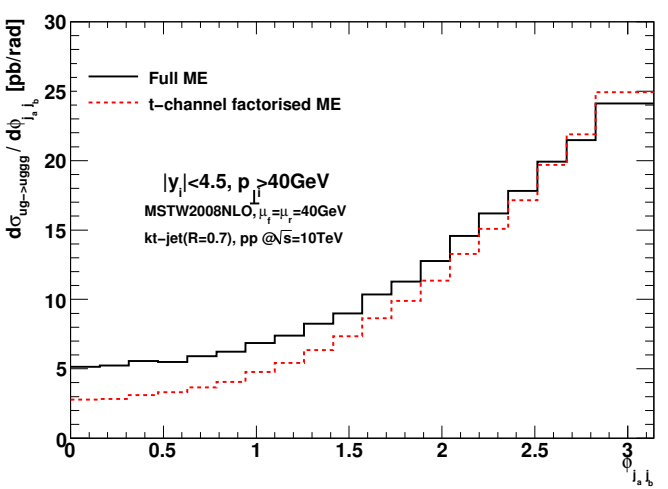

(d)

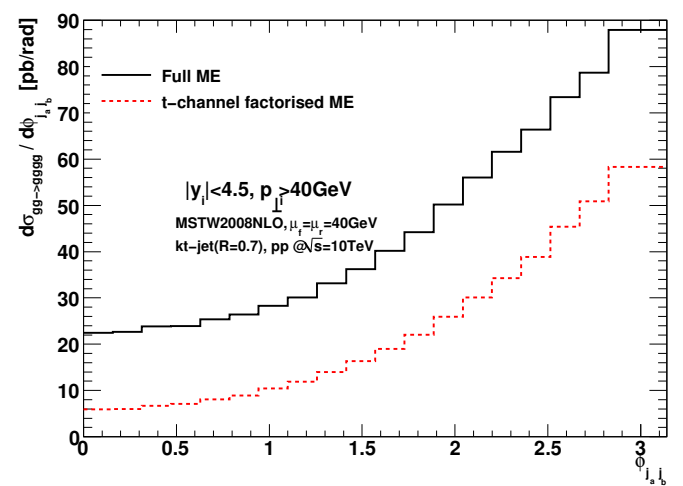

(f)

Figure 15. A comparison between the differential 4-jet cross section obtained using lowest order matrix elements obtained from Madgraph and the $t$-channel factorised formalism for the process (a)-(b) $u d \rightarrow u g g d$ with the $u$-quark incoming in the positive $z$-direction. (a) the rapidity difference between the most forward and backward jet (b) the distribution on the azimuthal angle between the most forward and backward jet. Similarly for $u g \rightarrow u g g g$ (c)-(d) and $g g \rightarrow g g g g$ (e)-(f).

blocks for the resummation.

We again illustrate the performance of the approximations by analysing the differential 


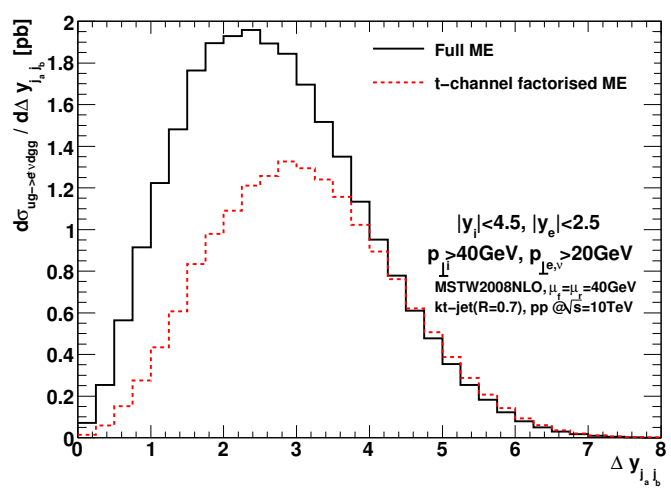

(a)

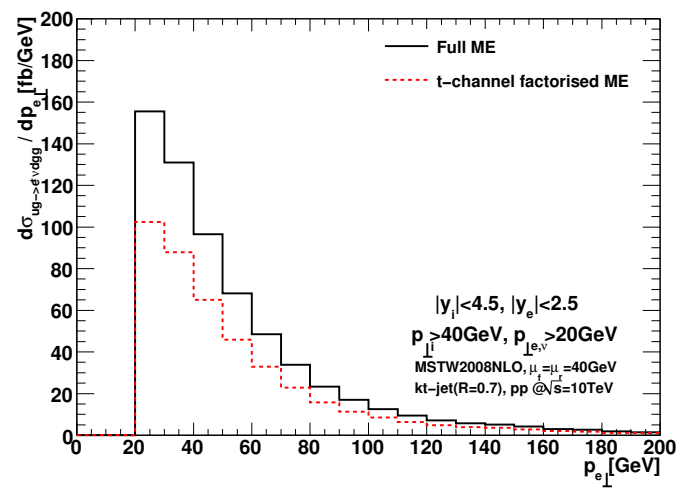

(c)

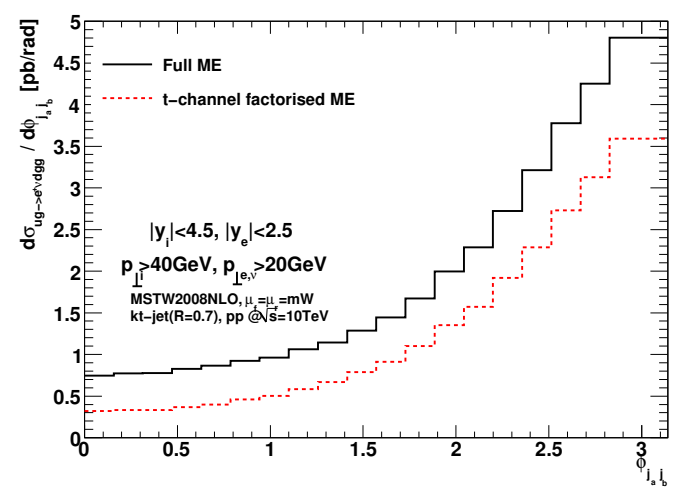

(b)

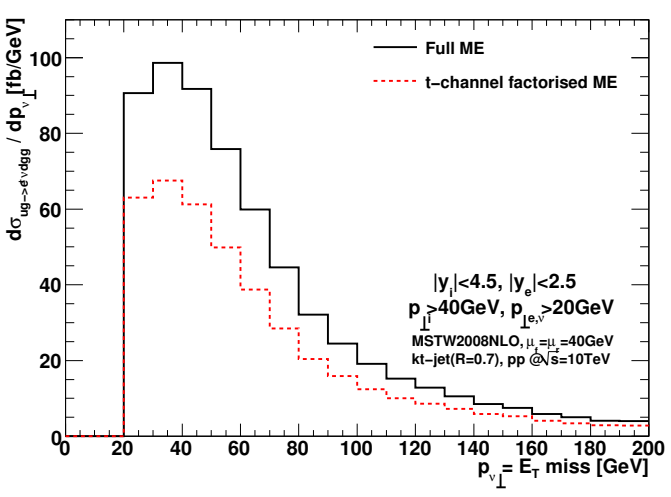

(d)

Figure 16. A comparison between the differential $W+3$-jet cross section obtained using lowest order matrix elements obtained from Madgraph and the $t$-channel factorised formalism for the process $u g \rightarrow e^{+} \nu d g g$ with the $u$-quark incoming in the positive $z$-direction. (a) the rapidity difference between the most forward and backward jet (b) the distribution on the azimuthal angle between the most forward and backward jet (c) the $p_{\perp}$ distribution of the electron and (d) the $p_{\perp}$ distribution of the neutrino, which is the missing transverse energy.

cross section with respect to the rapidity difference and azimuthal angle between the most forward and most backward jets in figure 16. Also shown are the $p_{\perp}$ distributions of both the electron and the neutrino. The latter is the distribution of missing transverse energy for these events.

The rapidity distribution is peaked slightly to the right in our formalism, as for the jets, and we still see that at larger rapidity differences the results converge. The shapes of the azimuthal angle, $p_{e \perp}$ and $p_{\nu \perp}$ distributions are very good although in each case, our formalism slightly underestimates the full matrix element, as was the case for the pure jet events. This is probably again due to collinear enhancements in the full matrix element, and the general underestimate of the $q g$-channel for smaller rapidities.

Figure 17 repeats these distributions now for the production of a $W$ boson in association with four jets. Again we see that the shapes of the distributions are reproduced quite well. 


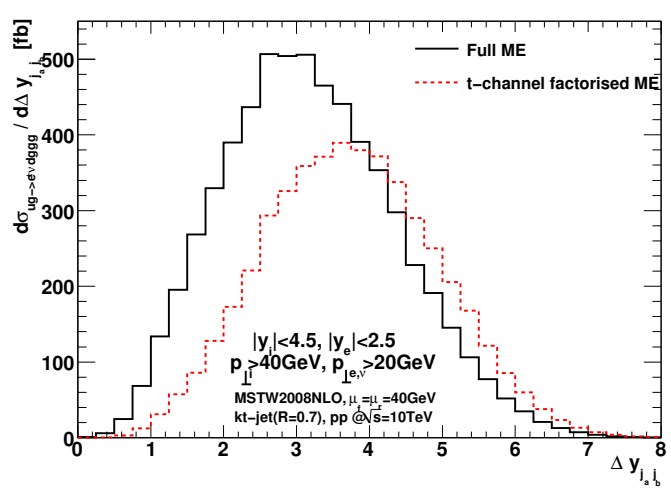

(a)

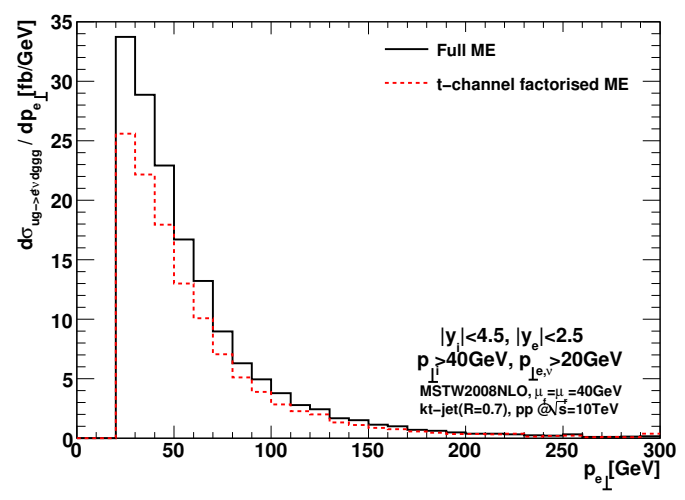

(c)

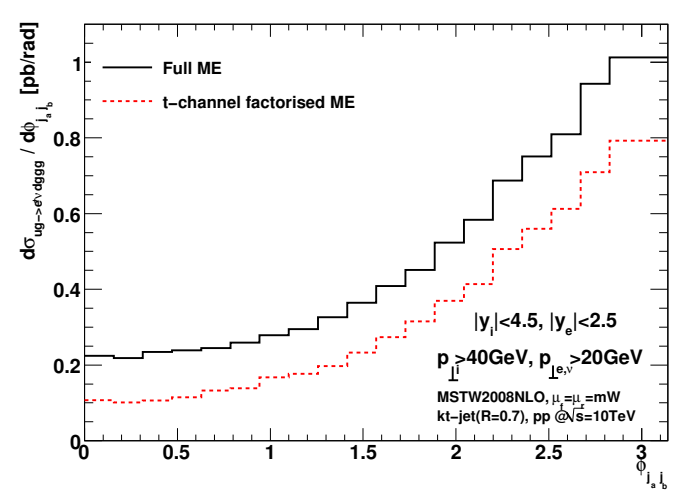

(b)

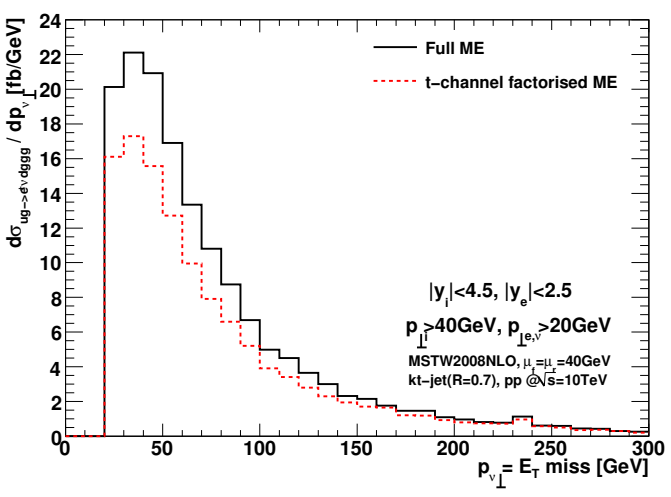

(d)

Figure 17. A comparison between the differential $W+4$-jet cross section obtained using lowest order matrix elements obtained from Madgraph and the $t$-channel factorised formalism for the process $u g \rightarrow e^{+} \nu d g g g$ with the $u$-quark incoming in the positive $z$-direction. (a) the rapidity difference between the most forward and backward jet (b) the distribution on the azimuthal angle between the most forward and backward jet (c) the $p_{\perp}$ distribution of the electron and (d) the $p_{\perp}$ distribution of the neutrino.

\section{$4.3 \mathrm{Z}+$ jets}

We now present similar results for the production of a $Z$ boson (decaying to charged leptons) in association with three (and four) jets, again for one of the dominant channels, $u g \rightarrow e^{+} e^{-} u g(g) g$, with the following set of cuts:

$$
\begin{array}{ll}
p_{j_{\perp}}>40 \mathrm{GeV} & p_{e \perp}>20 \mathrm{GeV} \\
\left|y_{j}\right|<4.5 & \left|y_{e}\right|<2.5
\end{array}
$$

The differential cross sections with respect to the rapidity difference, the azimuthal angle between the most forward and most backward jets and the transverse momentum of both the electron and anti-electron are all shown in figure 18.

The results resemble those for $W$ boson production. The slight difference between the $e^{+}$and $\nu$ transverse momentum distributions, figure 16, are not seen in figure 18 because identical cuts are now applied to both leptons here (the rapidity cut was not applied to the 


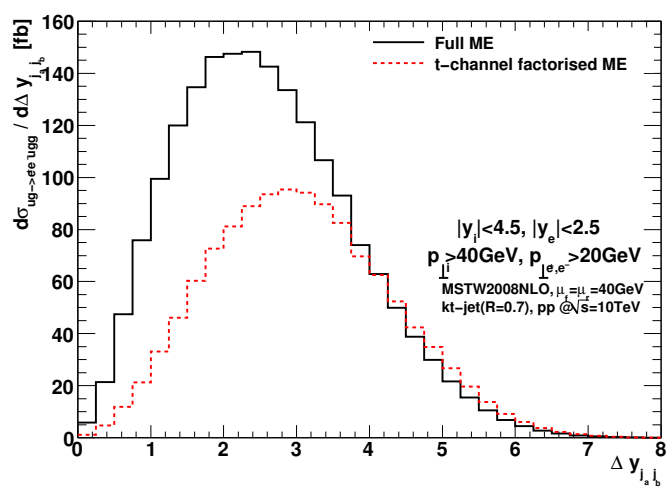

(a)

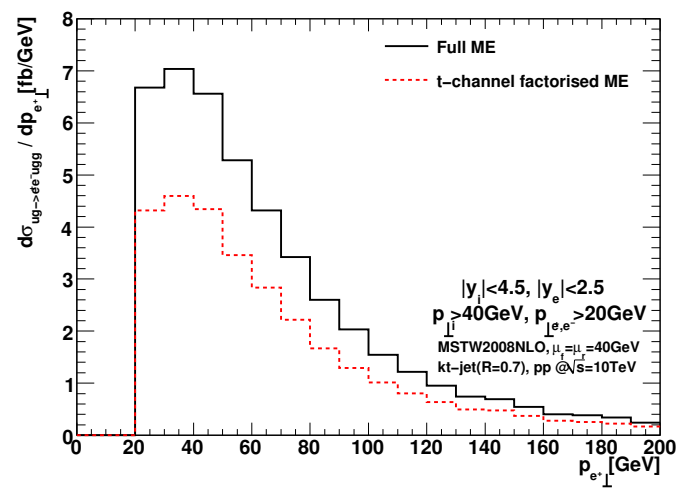

(c)

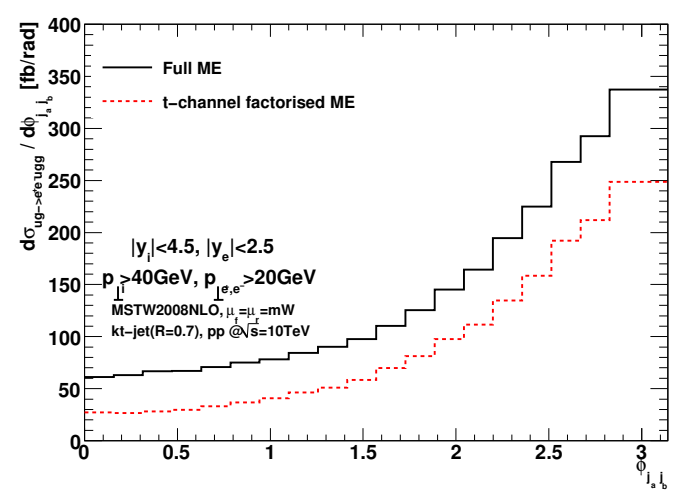

(b)

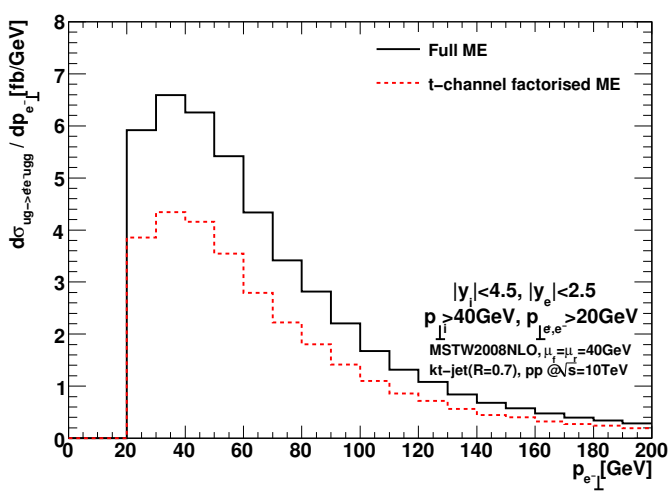

(d)

Figure 18. A comparison between the differential $Z+3$-jet cross section obtained using lowest order matrix elements obtained from Madgraph and the $t$-channel factorised formalism for the process $u g \rightarrow e^{+} e^{-} u g g$ with the $u$-quark incoming in the positive $z$-direction. (a) the rapidity difference between the most forward and backward jet (b) the distribution on the azimuthal angle between the most forward and backward jet (c) the $p_{\perp}$ distribution of the $e^{+}$and (d) the $p_{\perp}$ distribution of the $e^{-}$.

invisible neutrino for the $W$ events). The spin correlation of the $W$ to $e^{+}$is also washed out when it is replaced by a $Z$ boson.

Figure 19 repeats these distributions now for the production of a $Z$ boson in association with four jets. Again we see that the shapes of the distributions are reproduced very well by the simple approximations developed in section 3.3 .

\subsection{Higgs boson + jets}

In this section, we explore how well our new formalism (section 3.4) reproduces the results obtained using the full matrix element (at tree-level, where the fixed order results can be readily obtained). In the following analysis, we apply phase space cuts as follows:

$$
\begin{aligned}
p_{j_{\perp}} & >40 \mathrm{GeV} & y_{j a} \cdot y_{j b} & <0 \\
y_{j} & <4.5 & \left|y_{j a}-y_{j b}\right| & >2 \\
y_{j a} & \leq y_{h} \leq y_{j b} & &
\end{aligned}
$$




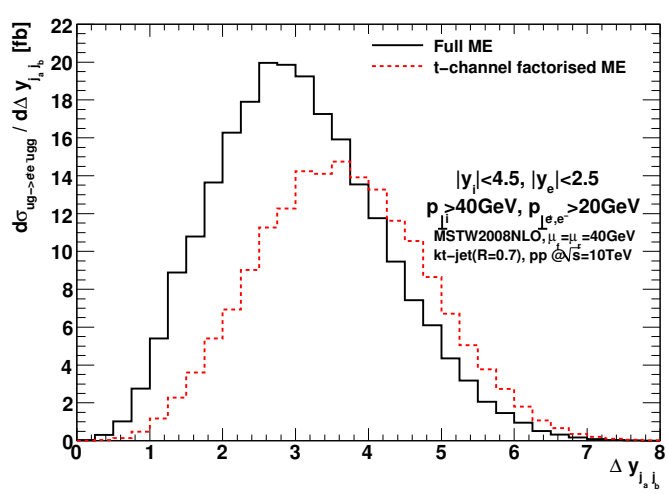

(a)

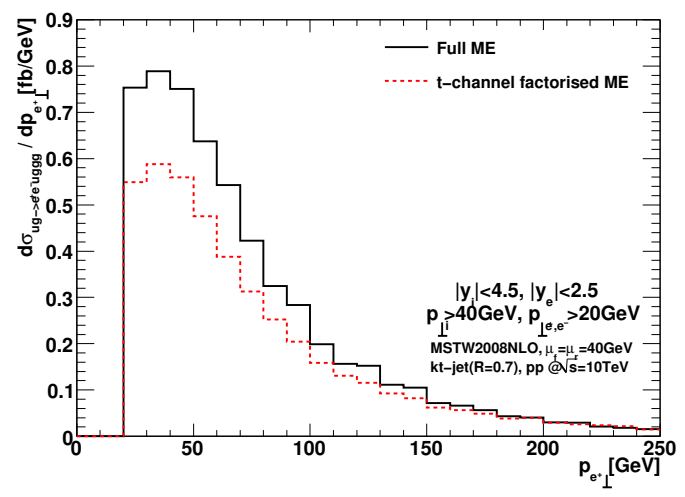

(c)

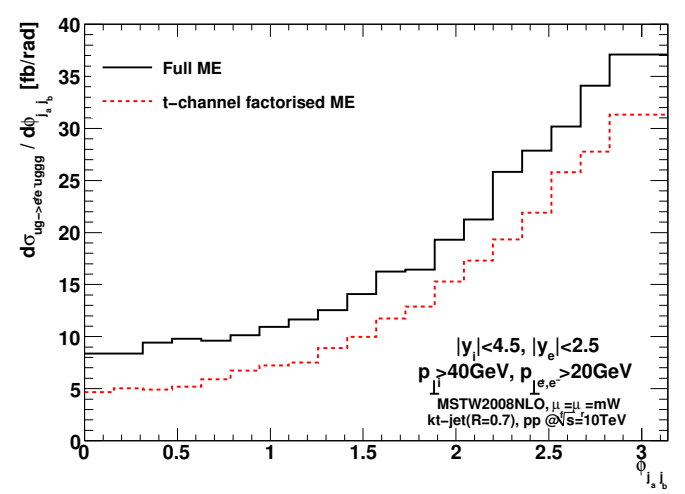

(b)

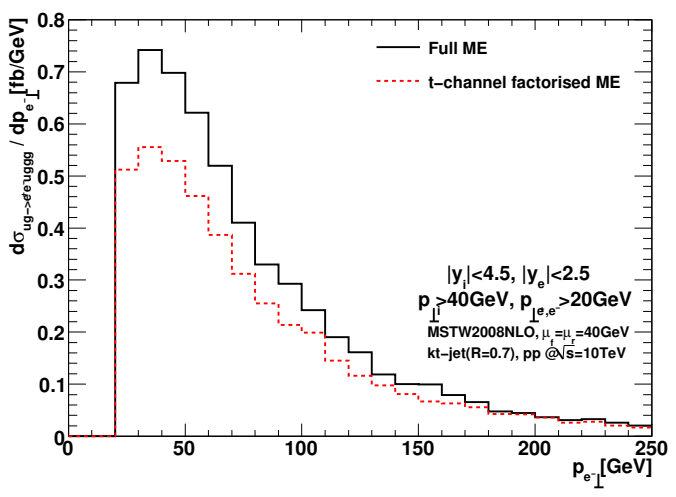

(d)

Figure 19. As in figure 18, but now for 4 jets in the final state: $u g \rightarrow e^{+} e^{-} u g g g$.

The merits of these cuts are discussed elsewhere [18, 19]. In figure 20 we compare the results obtained for the $h j j$-channel in four approximation:

1. Lowest order QCD

2. Lowest order QCD, but including only the flavour and rapidity configurations which are taking into account in the $t$-channel factorised framework

3. The results obtained using the tree-level (lowest order predictions) of the framework of ref. $[18,19]$

4. The results obtained using the tree-level (lowest order predictions) of the formalism of section 3.4

We start by noting that distributions and normalisation is by far dominated by the rapidity and flavour configurations of the final states which allow for a colour octet exchange between every rapidity-neighbours (these are the only contributions in the leading logarithmic approximation). The results obtained by summing over all final state configurations are indicated by the full lines, and the contribution from the "leading logarithmic" final states are indicated by the dash-dotted line. 


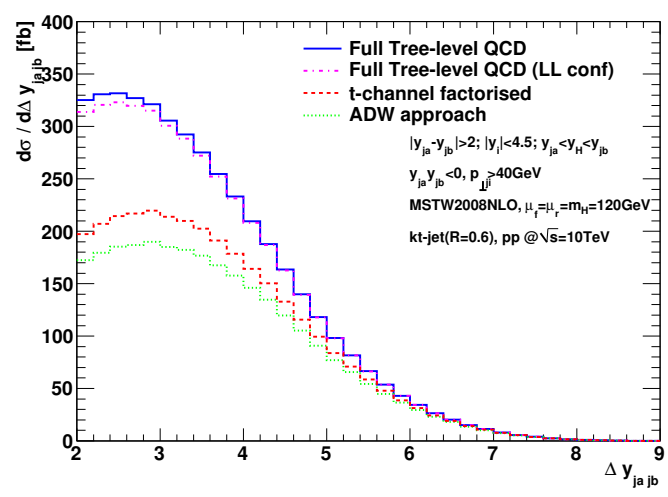

(a)

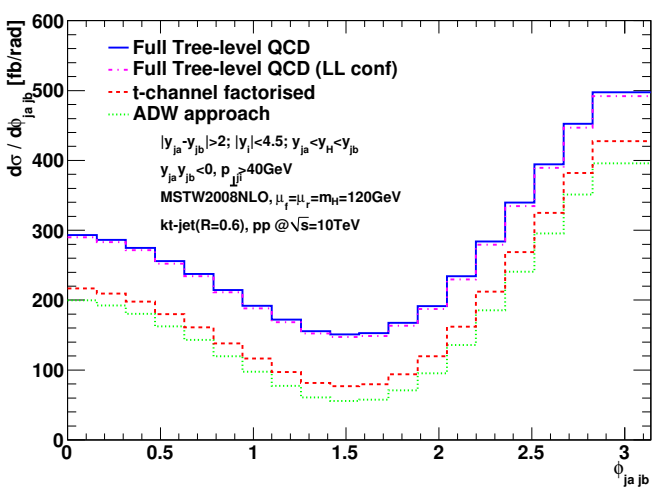

(b)

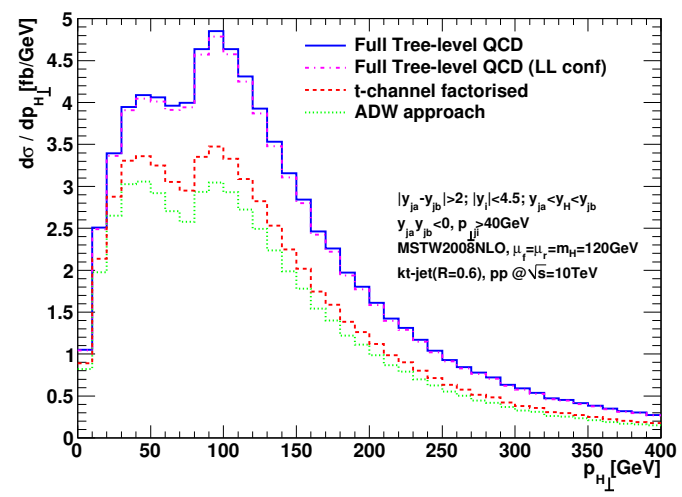

(c)

Figure 20. A comparison between the different $H+2$ jet cross sections using lowest order matrix elements obtained from MadGraph, the factorised approximation of $[18,19]$ and the formalism of section 3.4 for the sum over all relevant subprocesses $p p \rightarrow j H j$. For the results obtained using the full tree-level matrix elements, we have with the line marked "LL conf" indicated the impact of taking into account only the flavour and phase space configurations (rapidity orderings) which are approximated in the (leading logarithmic) $t$-channel factorised picture.

The difference between the results obtained with full QCD and in the $t$-channel factorised is by far dominated by the channels consisting of all gluons plus a Higgs boson. This is consistent with the observations made in figures 14-15. Also, we note that using full tree-level QCD, the distribution of the rapidity difference between the most forward and backward hard jet is peaked around 2.5 for Higgs boson production in association with two jets, and 3.5 for Higgs boson production in association with three jets. The position of the peaks are similar to the situation of pure jets, and highlights the universality of the QCD radiation pattern. The rapidity distribution obtained with the $t$-channel factorised matrix element peaks at slightly larger rapidities - this is simply because the gluon-gluon induced channel is underestimated at smaller rapidities; a feature also observed for pure jets.

We note that the formalism developed in the present study is a slight improvement over the earlier study in ref. $[18,19]$ in terms of reproducing the shape and normalisation of results obtained with full fixed order QCD. It should be noted that the results of the 


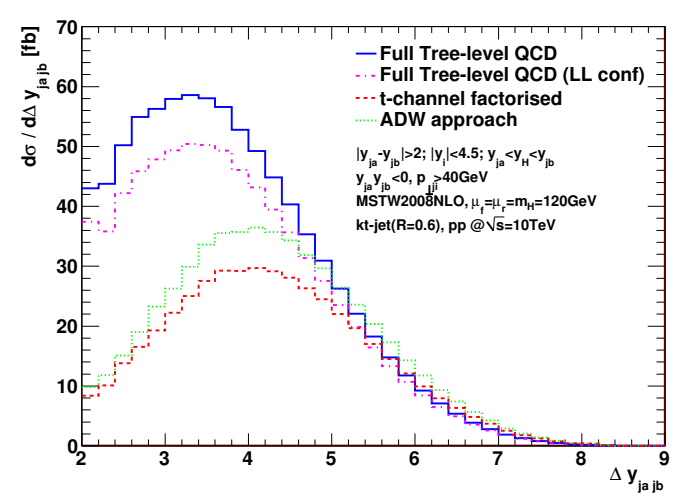

(a)

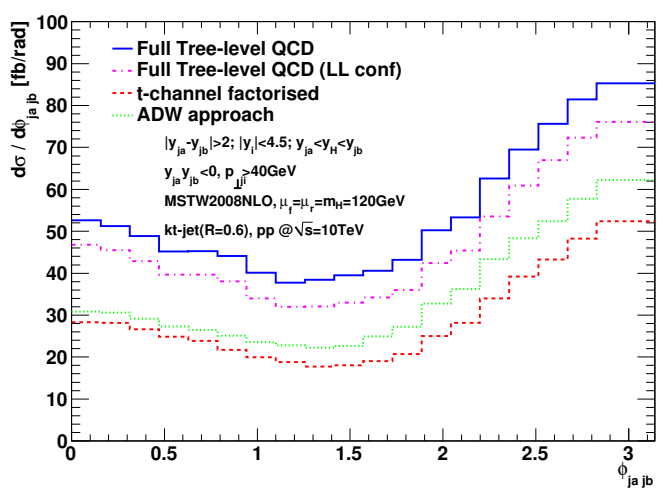

(b)

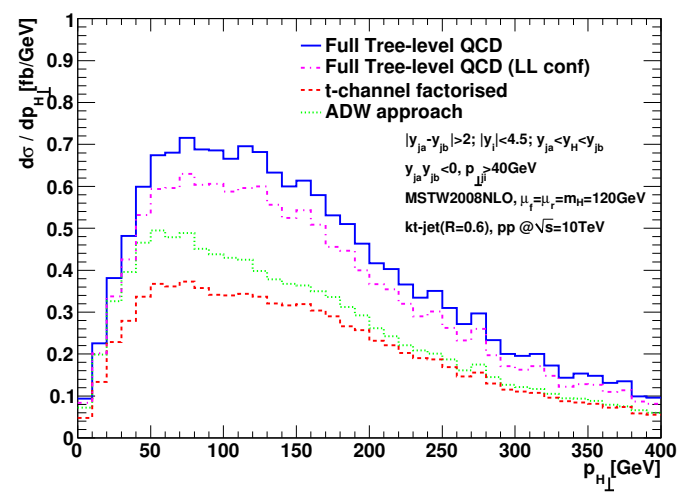

(c)

Figure 21. As in figure 20, but now for all subprocesses contributing to $p p \rightarrow H j j j$.

$t$-channel factorised approach are obtained more than 2 orders of magnitude faster than the results relying on the evaluation of the full matrix element with MadGraph [13] (all other components of the calculation are identical: phase space generation, pdfs etc.).

\section{Conclusions}

We have demonstrated the universal analytical structure of scattering amplitudes in the multi-Regge kinematic (MRK) limit of infinite rapidity separation between all produced particles for a range of scattering processes which are important for the LHC phenomenology (jets, $W+$ jets, $Z+$ jets and $H+$ jets). The universal behaviour in this limit is interpreted in terms of the scattering amplitudes being dominated by the poles in the $t$ channel momenta.

In section 3 we developed a formalism which is exact in the MRK limit, and fulfils the three requirement listed in section 3 necessary for constructing a relevant all-order summation: Inclusiveness, Simplicity and Accuracy. The formalism is inclusive in the sense that it captures the leading real and virtual corrections in the MRK limit. It is sufficiently simple that the cancellation of the infra-red poles can be organised explicitly to 
all orders (because of the simple structure of radiative corrections in the MRK limit). The results for the approximations to tree-level are obtained more than 2 orders of magnitudes faster than using standard tools for evaluating the full tree-level matrix element. This will allow for the all-order sum to be computed by explicit evaluation of the exclusive $n$-parton final states, and thus allow exclusive (or inclusive) analyses to be performed. Such an implementation will allow for matching to full tree-level results, where such are available.

We have shown examples of the results obtained in the framework for all 4 processes mentioned above, and compared to the results of using full leading order matrix elements. The results are very encouraging; the overall accuracy is good even without phase space cuts to enhance the accuracy of the approximation, and the discrepancies which do exists are limited to the region of small rapidity separation, where the first few orders in the perturbative series should be sufficient and the corrections can be included by matching. This is obviously necessary in order for the resummation, which will be built on the formalism, to be relevant for LHC phenomenology. The phenomenological implications of the resummation will be the focus of future studies.

\section{Acknowledgments}

JMS would like to thank the CERN theory group for kind hospitality at various stages of this project and is supported by the UK Science and Technology Facilities Council (STFC). This work was supported by the EC Marie-Curie Research Training Network "Tools and Precision Calculations for Physics Discoveries at Colliders" under contract MRTN-CT2006-035505.

\section{A Spinor representation}

We use the following representation for the spinors. For outgoing particles with 4-momentum $p, p^{ \pm}=E \pm p_{z}$ and $p_{\perp}=p_{x}+i p_{y}$, we use

$$
u^{+}(p)=\left(\begin{array}{c}
\sqrt{p^{+}} \\
\sqrt{p^{-}} \frac{p_{\perp}}{\left|p_{\perp}\right|} \\
0 \\
0
\end{array}\right) \quad \text { and } \quad u^{-}(p)=\left(\begin{array}{c}
0 \\
0 \\
\sqrt{p^{-}} \frac{p_{\perp}^{*}}{\mid p_{\perp}} \\
-\sqrt{p^{+}}
\end{array}\right) .
$$

For incoming particles with 4-momentum $p$ moving in the + direction, we use:

$$
u^{+}(p)=\left(\begin{array}{c}
\sqrt{p^{+}} \\
0 \\
0 \\
0
\end{array}\right) \quad \text { and } \quad u^{-}(p)=\left(\begin{array}{c}
0 \\
0 \\
0 \\
-\sqrt{p^{+}}
\end{array}\right) .
$$

For incoming particles with 4-momentum $p$ moving in the - direction, we use:

$$
u^{+}(p)=\left(\begin{array}{c}
0 \\
-\sqrt{p^{-}} \\
0 \\
0
\end{array}\right) \quad \text { and } \quad u^{-}(p)=\left(\begin{array}{c}
0 \\
0 \\
-\sqrt{p^{-}} \\
0
\end{array}\right) .
$$


We use the following representation for the gamma matrices:

$$
\begin{aligned}
& \gamma^{0}=\left(\begin{array}{llll}
0 & 0 & 1 & 0 \\
0 & 0 & 0 & 1 \\
1 & 0 & 0 & 0 \\
0 & 1 & 0 & 0
\end{array}\right), \quad \gamma^{1}=\left(\begin{array}{cccc}
0 & 0 & 0 & -1 \\
0 & 0 & -1 & 0 \\
0 & 1 & 0 & 0 \\
1 & 0 & 0 & 0
\end{array}\right), \\
& \gamma^{2}=\left(\begin{array}{cccc}
0 & 0 & 0 & i \\
0 & 0 & -i \\
0 & -i & 0 & 0 \\
i & 0 & 0 & 0
\end{array}\right), \quad \gamma^{3}=\left(\begin{array}{cccc}
0 & 0 & -1 & 0 \\
0 & 0 & 0 & 1 \\
1 & 0 & 0 & 0 \\
0 & -1 & 0 & 0
\end{array}\right) .
\end{aligned}
$$

We also use the shorthands

$$
\langle i j\rangle=\bar{u}^{-}\left(p_{i}\right) u^{+}\left(p_{j}\right) \quad \text { and } \quad[i j]=\bar{u}^{+}\left(p_{i}\right) u^{-}\left(p_{j}\right) .
$$

\section{B Momentum configurations}

Here we list, for completeness, the momentum configurations used for the plots of the matrix elements. Those for the pure jet events (figures 2 and 8 ) are given in the text. For the production of either a $W$ or $Z$ (decaying to $\ell \bar{\ell}$ ) in association with jets (figures $3,4,10$ and 12), we use the following momentum configurations. For 2 jets in the final state:

$$
\begin{aligned}
p_{i} & =\left(k_{\perp i} \cosh \left(y_{i}\right), k_{\perp i} \cos \left(\phi_{i}\right), k_{\perp i} \sin \left(\phi_{i}\right), k_{\perp i} \sinh \left(y_{i}\right)\right), \\
k_{\perp 1} & =k_{\perp \bar{\ell}}=40 \mathrm{GeV}, k_{\perp \ell}=\frac{m_{V}^{2}}{2 k_{\perp \bar{\ell}}\left(\cosh \left(y_{\bar{\ell}}-y_{\ell}\right)-\cos \left(\phi_{\bar{\ell}}-\phi_{\ell}\right)\right)}, \\
\phi_{1} & =\pi, \quad \phi_{\bar{\ell}}=\pi+0.2, \quad \phi_{\ell}=-(\pi+0.2), \\
y_{1} & =\Delta, \quad y_{2}=-\Delta, \quad y_{\bar{\ell}}=\Delta, \quad y_{\ell}=\Delta-1.5, \\
p_{2 \perp} & =-p_{1 \perp}-p_{\bar{\ell} \perp}-p_{\ell \perp .}
\end{aligned}
$$

For 3 jets in the final state:

$$
\begin{aligned}
p_{i} & =\left(k_{\perp i} \cosh \left(y_{i}\right), k_{\perp i} \cos \left(\phi_{i}\right), k_{\perp i} \sin \left(\phi_{i}\right), k_{\perp i} \sinh \left(y_{i}\right)\right), \\
k_{\perp 1} & =k_{\perp 2}=k_{\perp \bar{\ell}}=40 \mathrm{GeV}, \quad k_{\perp \ell}=\frac{m_{V}^{2}}{2 k_{\perp \bar{\ell}}\left(\cosh \left(y_{\bar{\ell}}-y_{\ell}\right)-\cos \left(\phi_{\bar{\ell}}-\phi_{\ell}\right)\right)}, \\
\phi_{1} & =2 \pi / 3, \quad \phi_{2}=0, \quad \phi_{\bar{\ell}}=\pi / 2, \quad \phi_{\ell}=-\pi / 2, \quad y_{\bar{\ell}}=\Delta, \quad y_{\ell}=\Delta-1.5, \\
y_{1} & =\Delta, \quad y_{2}=0, \quad y_{3}=-\Delta, \quad y, \quad \\
p_{3 \perp} & =-p_{1 \perp}-p_{2 \perp}-p_{\bar{\ell} \perp}-p_{\ell \perp},
\end{aligned}
$$

while the $4 j$ final state events use:

$$
\begin{aligned}
p_{i} & =\left(k_{\perp i} \cosh \left(y_{i}\right), k_{\perp i} \cos \left(\phi_{i}\right), k_{\perp i} \sin \left(\phi_{i}\right), k_{\perp i} \sinh \left(y_{i}\right)\right), \\
k_{\perp 1} & =k_{\perp 2}=k_{\perp 3}=k_{\perp \bar{\ell}}=40 \mathrm{GeV}, \quad k_{\perp \ell}=\frac{m_{V}^{2}}{2 k_{\perp \bar{\ell}}\left(\cosh \left(y_{\bar{\ell}}-y_{\ell}\right)-\cos \left(\phi_{\bar{\ell}}-\phi_{\ell}\right)\right)}, \\
\phi_{1} & =\pi, \quad \phi_{2}=\pi / 2, \quad \phi_{3}=-\pi / 3, \quad \phi_{\bar{\ell}}=\pi / 4, \quad \phi_{\ell}=-\pi / 4, \quad y_{\bar{\ell}}=\Delta, \quad y_{\ell}=\Delta, \\
y_{1} & =\Delta, \quad y_{2}=\Delta / 3, \quad y_{3}=-\Delta / 3, \quad y_{4}=-\Delta, \quad p, \quad \\
p_{4 \perp} & =-p_{1 \perp}-p_{2 \perp}-p_{3 \perp}-p_{\bar{\ell} \perp}-p_{\ell \perp} .
\end{aligned}
$$


For the production of a Higgs boson in association with jets (figures 5 and 13) we use the following momentum configurations. For 2 jets in the final state:

$$
\begin{aligned}
p_{1} & =(40 \sqrt{2} \cosh (\Delta),-40,40,40 \sqrt{2} \sinh (\Delta), \\
p_{H} & =\left(\sqrt{40^{2}+m_{H}^{2}}, 0,-40,0\right) \mathrm{GeV}, \\
p_{2} & =(40 \cosh (-\Delta), 40,0,40 \sinh (-\Delta)) \mathrm{GeV},
\end{aligned}
$$

and for 3 jets in the final state:

$$
\begin{aligned}
p_{1} & =(40 \cosh (\Delta),-40,0,40 \sinh (\Delta), \\
p_{H} & =\left(\sqrt{40^{2}+m_{H}^{2}} \cosh (\Delta / 3), 0,-40, \sqrt{40^{2}+m_{H}^{2}} \sinh (\Delta / 3)\right) \mathrm{GeV}, \\
p_{2} & =(40 \cosh (-\Delta / 3), 0,40,40 \sinh (-\Delta / 3)) \mathrm{GeV}, \\
p_{3} & =(40 \cosh (-\Delta), 40,0,40 \sinh (-\Delta)) \mathrm{GeV} .
\end{aligned}
$$

Open Access. This article is distributed under the terms of the Creative Commons Attribution Noncommercial License which permits any noncommercial use, distribution, and reproduction in any medium, provided the original author(s) and source are credited.

\section{References}

[1] T. Sjöstrand, S. Mrenna and P. Skands, A Brief Introduction to PYTHIA 8.1, Comput. Phys. Commun. 178 (2008) 852 [arXiv:0710.3820] [SPIRES].

[2] M. Bahr et al., HERWIG++ Physics and Manual, Eur. Phys. J. C 58 (2008) 639 [arXiv: 0803.0883] [SPIRES].

[3] T. Gleisberg et al., Event generation with SHERPA 1.1, JHEP 02 (2009) 007 [arXiv:0811.4622] [SPIRES].

[4] S. Catani, F. Krauss, R. Kuhn and B.R. Webber, QCD Matrix Elements + Parton Showers, JHEP 11 (2001) 063 [hep-ph/0109231] [SPIRES].

[5] L. Lönnblad, ARIADNE version 4: A Program for simulation of QCD cascades implementing the color dipole model, Comput. Phys. Commun. 71 (1992) 15 [SPIRES].

[6] S. Frixione and B.R. Webber, Matching NLO QCD computations and parton shower simulations, JHEP 06 (2002) 029 [hep-ph/0204244] [SPIRES].

[7] S. Frixione, P. Nason and G. Ridolfi, The POWHEG-hvq manual version 1.0, arXiv:0707.3081 [SPIRES].

[8] L.N. Lipatov, The parton model and perturbation theory, Sov. J. Nucl. Phys. 20 (1975) 94 [SPIRES].

[9] V.S. Fadin, E.A. Kuraev and L.N. Lipatov, On the Pomeranchuk Singularity in Asymptotically Free Theories, Phys. Lett. B 60 (1975) 50 [SPIRES].

[10] E.A. Kuraev, L.N. Lipatov and V.S. Fadin, The Pomeranchuk Singularity in Nonabelian Gauge Theories, Sov. Phys. JETP 45 (1977) 199 [SPIRES].

[11] E.A. Kuraev, L.N. Lipatov and V.S. Fadin, Multi-Reggeon Processes in the Yang-Mills Theory, Sov. Phys. JETP 44 (1976) 443 [SPIRES]. 
[12] B.L. Combridge and C.J. Maxwell, Untangling Large $p_{T}$ Hadronic Reactions, Nucl. Phys. B 239 (1984) 429 [SPIRES].

[13] J. Alwall et al., MadGraph/MadEvent v4: The New Web Generation, JHEP 09 (2007) 028 [arXiv:0706.2334] [SPIRES].

[14] J.R. Andersen, V. Del Duca, F. Maltoni and W.J. Stirling, $W$ boson production with associated jets at large rapidities, JHEP 05 (2001) 048 [hep-ph/0105146] [SPIRES].

[15] V. Del Duca, W. Kilgore, C. Oleari, C.R. Schmidt and D. Zeppenfeld, Kinematical limits on Higgs boson production via gluon fusion in association with jets, Phys. Rev. D 67 (2003) 073003 [hep-ph/0301013] [SPIRES].

[16] V.S. Fadin, R. Fiore, M.G. Kozlov and A.V. Reznichenko, Proof of the multi-Regge form of QCD amplitudes with gluon exchanges in the NLA, Phys. Lett. B 639 (2006) 74 [hep-ph/0602006] [SPIRES].

[17] A.V. Bogdan and V.S. Fadin, A proof of the reggeized form of amplitudes with quark exchanges, Nucl. Phys. B $\mathbf{7 4 0}$ (2006) 36 [hep-ph/0601117] [SPIRES].

[18] J.R. Andersen and C.D. White, A New Framework for Multijet Predictions and its application to Higgs Boson production at the LHC, Phys. Rev. D 78 (2008) 051501 [arXiv: 0802.2858] [SPIRES].

[19] J.R. Andersen, V. Del Duca and C.D. White, Higgs Boson Production in Association with Multiple Hard Jets, JHEP 02 (2009) 015 [arXiv: 0808.3696] [SPIRES].

[20] V. Del Duca, A. Frizzo and F. Maltoni, Factorization of tree QCD amplitudes in the high-energy limit and in the collinear limit, Nucl. Phys. B 568 (2000) 211 [hep-ph/9909464] [SPIRES].

[21] V.S. Fadin, M.G. Kozlov and A.V. Reznichenko, Radiative corrections to QCD amplitudes in quasi-multi-Regge kinematics, Phys. Atom. Nucl. 67 (2004) 359 [hep-ph/0302224] [SPIRES].

[22] V.S. Fadin, The gluon Reggeization in perturbative QCD at NLO, hep-ph/0511121 [SPIRES].

[23] C.R. Schmidt, A Monte Carlo Solution to the BFKL Equation, Phys. Rev. Lett. 78 (1997) 4531 [hep-ph/9612454] [SPIRES].

[24] L.H. Orr and W.J. Stirling, Dijet production at hadron hadron colliders in the BFKL approach, Phys. Rev. D 56 (1997) 5875 [hep-ph/9706529] [SPIRES].

[25] J.R. Andersen and A. Sabio Vera, The gluon Green's function in the BFKL approach at nextto-leading logarithmic accuracy, Nucl. Phys. B 679 (2004) 345 [hep-ph/0309331] [SPIRES].

[26] J.R. Andersen, On the role of NLL corrections and energy conservation in the high energy evolution of QCD, Phys. Lett. B 639 (2006) 290 [hep-ph/0602182] [SPIRES].

[27] A.D. Martin, W.J. Stirling, R.S. Thorne and G. Watt, Parton distributions for the LHC, Eur. Phys. J. C 63 (2009) 189 [arXiv:0901.0002] [SPIRES].

[28] M. Cacciari and G.P. Salam, Dispelling the $N^{3}$ myth for the $k_{t}$ jet-finder, Phys. Lett. B 641 (2006) 57 [hep-ph/0512210] [SPIRES]. 